\title{
44. INORGANIC MAJOR, MINOR, AND TRACE ELEMENT GEOCHEMISTRY AND CLAY MINERALOGY OF SEDIMENTS FROM THE DEEP SEA DRILLING PROJECT LEG 96, GULF OF MEXICO'
}

\author{
Kevin T. Pickering, University of London \\ and \\ Dorrik A. V. Stow, University of Edinburgh ${ }^{2}$
}

\begin{abstract}
Sediment samples collected at DSDP Leg 96 Mississippi Fan Sites 615, 616, 620, 621, and 623, Orca Basin Site 618, and Pigmy Basin Site 619 were analyzed for 22 major, minor, and trace elements. This study was undertaken to document the downhole variability in inorganic geochemistry between sites. The mineralogy of the clays, including those from Sites 614,617 , and 622 on the fan, was determined by X-ray diffraction to define the principal clay minerals present at the sites, examine any downhole trends in clay mineralogy, and aid in the interpretation of the geochemical signature of the sediments. Clay mineral composition at all the sites is smectite:illite:chlorite:kaolinite in the approximate percentage ratio 50:20:20:10. Geochemical results indicate only slight variation between and within the sites, with the exception of a discrete unit of carbonates that occurs near the bottom of Site 615. Variation in the major, minor, and trace element composition can be explained by a change in the relative abundance of quartz, clay minerals, and carbonates.
\end{abstract}

\section{INTRODUCTION}

Eight hundred and twenty-eight samples were collected for inorganic geochemical analyses at the 11 Deep Sea Drilling Project (DSDP) sites visited on Leg 96 (Fig. 1). From this population, 145 samples from Sites $615,616,620,621$, and 623 on the Mississippi Fan, Site 618 in Orca Basin, and Site 619 in Pigmy Basin were analyzed for 22 major, minor, and trace elements (Table 1) and (in addition to samples from fan Sites 614, 617, and 622) for clay mineralogy (Table 2). This pilot study was undertaken to document, by sampling at regular downhole intervals, the general inorganic geochemistry of the Pleistocene Mississippi Fan sediments (with a cursory study of the intraslope basin sites for comparative purposes) and to relate this inorganic geochemical data to clay and smear-slide mineralogy.

\section{METHODS}

Samples collected for inorganic geochemical studies were air dried at $105^{\circ} \mathrm{C}$ and then ground in an agate ball mill for $15 \mathrm{~min}$. We diluted $0.5 \mathrm{~g}$ of the ground sample to $250 \mathrm{ml}$ using the lithium metaborate fusion technique for major-element analysis (Walsh and Howie, 1980) and $0.5 \mathrm{~g}$ of the sample was dissolved in $50 \mathrm{ml}$ using the hydrofluoric perchloric acid digestion technique for minor- and trace-element analysis (Walsh and Howie, 1980). The solutions were analyzed with the Phillips PV8210/ICP Emission Spectrometer at Kings College, University of London. The data were calibrated using igneous and sedimentary U.S. Geological Survey standards GSP-1, BHVO-1, AGV-1, SDC-1, MAG-1, QLO-1, SCo-1, and STM-1. Total iron ( $\left.\mathrm{FeO}+\mathrm{Fe}_{2} \mathrm{O}_{3}\right)$ was determined without analyzing for the different oxidation states.

For clay mineralogy analyses, the $<4 \mu \mathrm{m}$ fine fraction was separated by ultrasonic treatment and settling. Wherever possible the sam-

\footnotetext{
${ }^{1}$ Bouma, A. H., Coleman, J. M., Meyer, A. W., et al., Init. Repts. DSDP, 96: Washington (U.S. Govt. Printing Office).

2 Addresses: (Pickering, present address) Department of Geology, University of Leicester, Leicester LEI 7RH, United Kingdom; (Stow, present address) Department of Geology, University of Nottingham, Nottingham NG7 2RD, United Kingdom.
}

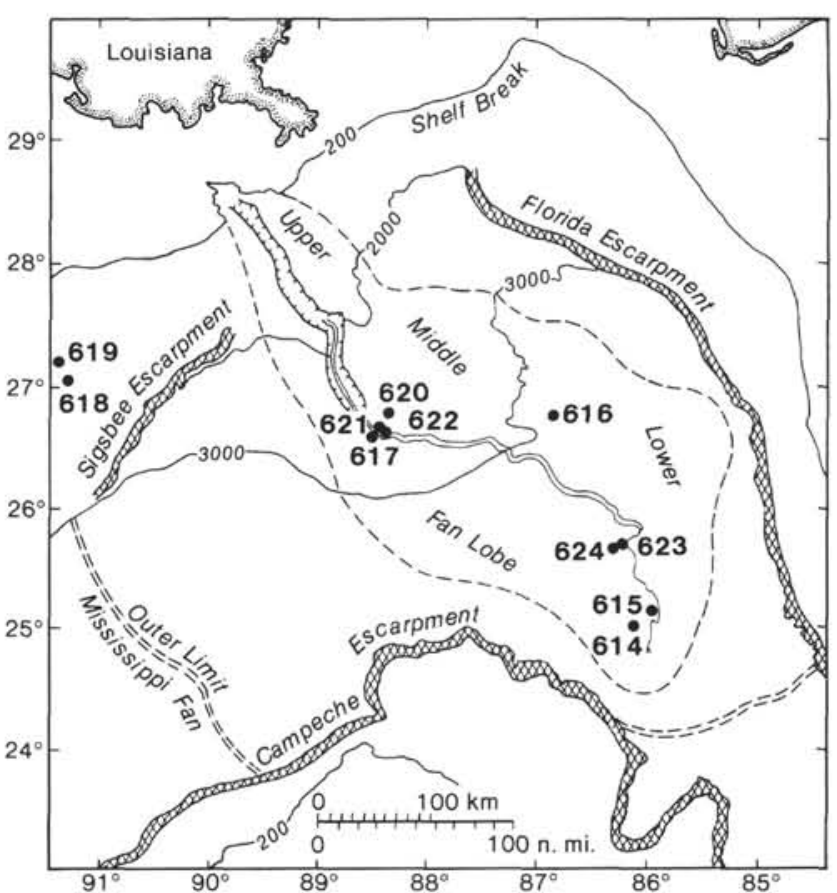

Figure 1. Location map of the sites drilled on DSDP Leg 96.

ples were split from those used to determine the inorganic geochemistry. Oriented aggregates were prepared on glass slides. Both untreated and glycolated (overnight at $60^{\circ} \mathrm{C}$ ) X-ray diffraction (XRD) traces were run from 4 to $40^{\circ} 2 \theta$ for all the samples; a third slow scan run was made, only for selected samples, to separate the kaolinite and chlorite peaks between 24 and $26^{\circ} 2 \theta$. Focused $\mathrm{CuK} \alpha$ radiation was used on all three scans at a scan speed of $1^{\circ} 2 \theta$ per minute. The XRD work was done at Edinburgh University. All mineral identification was made visually on the basis of known d-spacings (Carver, 1971).

The main clay minerals recognized were kaolinite, illite, chlorite, smectite, and an irregular mixed-layer species. Other identified minerals included quartz, feldspars, calcite, and dolomite. Peak heights 


\begin{tabular}{|c|c|c|c|c|c|c|c|c|c|c|c|c|c|c|c|c|c|c|c|c|c|c|c|}
\hline \multirow{2}{*}{$\begin{array}{c}\text { Core-Section } \\
\text { (interval in cm) }\end{array}$} & \multirow{2}{*}{$\begin{array}{l}\text { Sub-bottom } \\
\text { depth (m) }\end{array}$} & \multicolumn{10}{|c|}{ Major elements (wt.\%) } & \multicolumn{12}{|c|}{ Trace elements (ppm) } \\
\hline & & $\mathrm{SiO}_{2}$ & $\mathrm{Al}_{2} \mathrm{O}_{3}$ & $\mathrm{FeO} / \mathrm{Fe}_{2} \mathrm{O}_{3}$ & $\mathrm{MgO}$ & $\mathrm{CaO}$ & $\mathrm{Na}_{2} \mathrm{O}$ & $\mathrm{K}_{2} \mathrm{O}$ & $\mathrm{TiO}_{2}$ & $\mathrm{P}_{2} \mathrm{O}_{5}$ & $\mathrm{MnO}$ & $\mathrm{Ba}$ & $\mathrm{Ce}$ & Co & $\mathrm{Cr}$ & $\mathrm{Cu}$ & La & $\mathrm{Li}$ & $\mathrm{Ni}$ & Sc & $\mathrm{Sr}$ & $\mathrm{v}$ & $\mathrm{Y}$ \\
\hline \multicolumn{24}{|l|}{ Hole 615} \\
\hline $1-1,60.0-65.0$ & 0.63 & 51.83 & 15.57 & 6.37 & 3.08 & 2.99 & 2.68 & 2.83 & 0.67 & 0.16 & 0.12 & 497 & 70 & 15 & 116 & 35 & 46 & 55 & 54 & 15 & 143 & 161 & 29 \\
\hline $3-2,55.0-59.0$ & 14.17 & 37.77 & 16.34 & 7.16 & 3.10 & 3.15 & 2.08 & 2.77 & 0.65 & 0.17 & 0.13 & 513 & 70 & 15 & 128 & 38 & 46 & 58 & 54 & 16 & 151 & 174 & 30 \\
\hline $3-3,30.0-35.0$ & 15.43 & 55.45 & 9.98 & 3.66 & 2.53 & 4.29 & 1.79 & 2.02 & 0.46 & 0.11 & 0.08 & 559 & 49 & 12 & 88 & 21 & 30 & 29 & 40 & 8 & 163 & 79 & 20 \\
\hline $4-3,31.0-36.0$ & 23.43 & 86.09 & 6.62 & 1.93 & 1.06 & 2.68 & 1.69 & 1.57 & 0.30 & 0.05 & 0.04 & 571 & 34 & 7 & 47 & 8 & 22 & 11 & 21 & 4 & 196 & 34 & 13 \\
\hline $5-4,62.0-66.0$ & 38.14 & 57.16 & 14.26 & 5.66 & 3.00 & 4.26 & 1.74 & 2.34 & 0.61 & 0.15 & 0.14 & 557 & 58 & 15 & 123 & 33 & 38 & 50 & 51 & 13 & 160 & 168 & 25 \\
\hline $6-2,3.0-8.0$ & 44.06 & 61.52 & 6.77 & 3.08 & 3.74 & 7.03 & 1.97 & 1.91 & 0.47 & 0.12 & 0.10 & 588 & 51 & 12 & 95 & 23 & 27 & 25 & 43 & 7 & 187 & 74 & 20 \\
\hline $7-6,48.0-54.0$ & 56.41 & 80.40 & 6.88 & 2.20 & 1.70 & 3.92 & 1.76 & 1.65 & 0.33 & 0.06 & 0.05 & 612 & 40 & 8 & 50 & 11 & 24 & 12 & 26 & 4 & 199 & 39 & 15 \\
\hline $8-2,24.0-26.0$ & 59.45 & 75.09 & 11.18 & 3.75 & 3.02 & 4.91 & 1.93 & 2.34 & 0.47 & 0.11 & 0.09 & 619 & 52 & 12 & 100 & 26 & 31 & 33 & 41 & 9 & 182 & 96 & 21 \\
\hline $9-2,37.0-42.0$ & 69.21 & 74.02 & 8.26 & 2.17 & 2.22 & 4.33 & 2.16 & 2.11 & 0.33 & 0.09 & 0.05 & 680 & 41 & 8 & 54 & 13 & 24 & 16 & 26 & 5 & 207 & 43 & 16 \\
\hline $9-3,56.0-61.0$ & 70.90 & 58.79 & 16.28 & 6.53 & 3.02 & 3.89 & 1.72 & 2.59 & 0.65 & 0.17 & 0.11 & 585 & 49 & 15 & 140 & 36 & 37 & 54 & 56 & 15 & 143 & 194 & 25 \\
\hline $12-1,50.0-55.0$ & 96.70 & 61.24 & 13.87 & 5.06 & 3.07 & 4.85 & 1.53 & 3.05 & 0.66 & 0.10 & 0.10 & 1217 & 72 & 18 & 77 & 31 & 34 & 55 & 55 & 14 & 168 & 133 & 25 \\
\hline $18-1,93.0-95.0$ & 153.40 & 76.49 & 8.31 & 2.30 & 1.61 & 3.28 & 1.81 & 2.02 & 0.35 & 0.08 & 0.05 & 632 & 41 & 8 & 63 & 15 & 26 & 17 & 28 & 5 & 198 & 50 & 17 \\
\hline $22-4,62.0-64.0$ & 195.83 & 60.76 & 10.85 & 3.39 & 2.29 & 4.10 & 1.79 & 2.30 & 0.42 & 0.11 & 0.08 & 603 & 52 & 11 & 93 & 23 & 31 & 27 & 37 & 8 & 192 & 84 & 21 \\
\hline $29-1,128.0-130.0$ & 257.89 & 71.42 & 8.24 & 2.63 & 2.50 & 5.11 & 1.68 & 2.05 & 0.40 & 0.08 & 0.07 & 621 & 44 & 11 & 89 & 20 & 26 & 20 & 35 & 6 & 188 & 61 & 18 \\
\hline $29-5,70.0-72.0$ & 263.91 & 85.60 & 5.96 & 1.74 & 1.24 & 3.11 & 1.49 & 1.55 & 0.29 & 0.03 & 0.04 & 536 & 37 & 9 & 47 & 10 & 23 & 9 & 22 & 3 & 172 & 32 & 13 \\
\hline $32-1,68.0-70.0$ & 286.28 & 68.32 & 7.18 & 1.94 & 1.99 & 4.52 & 1.50 & 1.72 & 0.29 & 0.06 & 0.06 & 507 & 41 & 9 & 60 & 14 & 21 & 15 & 28 & 4 & 174 & 42 & 14 \\
\hline $34-1,130.0-135.0$ & 315.53 & 66.50 & 10.37 & 3.12 & 2.72 & 5.23 & 1.95 & 2.41 & 0.46 & 0.11 & 0.12 & 606 & 53 & 12 & 78 & 25 & 31 & 31 & 43 & 7 & 204 & 75 & 20 \\
\hline $38-1,51.0-53.0$ & 352.72 & 55.33 & 9.12 & 2.80 & 2.77 & 5.40 & 2.08 & 2.16 & 0.42 & 0.10 & 0.10 & 583 & 47 & 9 & 72 & 19 & 26 & 23 & 32 & 6 & 198 & 61 & 19 \\
\hline $43-1,40.0-45.0$ & 409.63 & 77.80 & 6.85 & 1.88 & 1.64 & 3.90 & 1.62 & 1.72 & 0.30 & 0.04 & 0.04 & 561 & 37 & 8 & 53 & 10 & 21 & 12 & 24 & 4 & 183 & 35 & 13 \\
\hline $43-3,80.0-85.0$ & 412.63 & 57.72 & 7.49 & 1.83 & 2.05 & 4.22 & 1.89 & 1.94 & 0.32 & 0.06 & 0.04 & 559 & 36 & 8 & 46 & 13 & 21 & 15 & 24 & 4 & 184 & 38 & 14 \\
\hline $46, \mathrm{CC}(11.0-14.0)$ & 428.58 & 61.35 & 9.17 & 3.18 & 2.79 & 5.15 & 1.69 & 2.13 & 0.40 & 0.09 & 0.07 & 533 & 45 & 15 & 110 & 24 & 27 & 27 & 53 & 8 & 180 & 71 & 20 \\
\hline $49-2,46.0-51.0$ & 477.68 & 13.68 & 3.92 & 1.51 & 1.70 & 39.02 & 0.89 & 0.69 & 0.17 & 0.08 & 0.07 & 160 & 40 & 14 & 31 & 28 & 0 & 23 & 42 & 6 & 2175 & 41 & 17 \\
\hline $50-3,80.0-85.0$ & 489.03 & 11.63 & 2.72 & 1.05 & 1.60 & 40.41 & 0.90 & 0.52 & 0.12 & 0.06 & 0.05 & 116 & 36 & 13 & 18 & 24 & 0 & 15 & 36 & 4 & 2156 & 29 & 14 \\
\hline $50-6,80.0-85.0$ & 493.53 & 16.75 & 3.02 & 1.09 & 1.65 & 37.68 & 1.05 & 0.68 & 0.13 & 0.06 & 0.04 & 137 & 35 & 13 & 23 & 24 & 0 & 15 & 38 & 4 & 1969 & 28 & 14 \\
\hline $50, \mathrm{CC}(20.0-25.0)$ & 494.60 & 19.93 & 3.20 & 1.15 & 1.60 & 36.72 & 0.96 & 0.73 & 0.15 & 0.06 & 0.04 & 163 & 34 & 12 & 22 & 23 & 0 & 13 & 36 & 4 & 1894 & 28 & 13 \\
\hline $51-2,28.0-30.0$ & 496.49 & 27.31 & 3.22 & 1.28 & 1.59 & 35.19 & 0.97 & 0.77 & 0.17 & 0.08 & 0.04 & 440 & 35 & 12 & 33 & 21 & 0 & 12 & 36 & 4 & 1847 & 27 & 13 \\
\hline $52-1,23.0-28.0$ & 504.46 & 20.70 & 4.35 & 1.63 & 1.02 & 40.68 & 1.18 & 0.73 & 0.18 & 0.05 & 0.11 & 162 & 42 & 14 & 5 & 24 & 0 & 20 & 42 & 6 & 1300 & 37 & 15 \\
\hline \multicolumn{24}{|l|}{ Hole 616} \\
\hline $1-2,45.0-50.0$ & 1.98 & 60.74 & 16.30 & 5.60 & 3.21 & 3.15 & 2.79 & 2.91 & 0.64 & 0.13 & 0.10 & 500 & 64 & 15 & 105 & 41 & 36 & 68 & 56 & 16 & 137 & 165 & 26 \\
\hline $1-3,45.0-50.0$ & 3.47 & 61.87 & 15.88 & 5.34 & 3.21 & 3.48 & 2.79 & 2.86 & 0.58 & 0.14 & 0.10 & 499 & 62 & 14 & 83 & 37 & 34 & 65 & 53 & 15 & 140 & 143 & 24 \\
\hline $2-5,20.0-24.0$ & 12.22 & 47.65 & 14.36 & 4.99 & 3.21 & 4.61 & 2.12 & 2.55 & 0.60 & 0.13 & 0.13 & 531 & 62 & 14 & 86 & 40 & 33 & 55 & 55 & 14 & 154 & 146 & 23 \\
\hline $3-4,50.0-54.0$ & 20.62 & 52.20 & 15.34 & 5.39 & 3.17 & 4.17 & 2.12 & 2.62 & 0.63 & 0.13 & 0.12 & 523 & 63 & 14 & 109 & 42 & 34 & 58 & 57 & 15 & 150 & 157 & 25 \\
\hline $4-2,100.0-105.0$ & 27.63 & 58.34 & 15.25 & 5.3 & 3.10 & 4.98 & 1.81 & 2.75 & 0.61 & 0.14 & 0.1 & 465 & 67 & 15 & 101 & 39 & 35 & 62 & 59 & 14 & 170 & 166 & 26 \\
\hline $5-3,37.0-40.0$ & 37.99 & 56.46 & 15.59 & 5.12 & 3.00 & 3.53 & 1.82 & 2.64 & 0.62 & 0.13 & 0.14 & 527 & 66 & 14 & 107 & 33 & 35 & 56 & 56 & 15 & 148 & 163 & 25 \\
\hline $6-4,100.0-105.0$ & 49.72 & 59.17 & 14.63 & 5.07 & 3.05 & 4.93 & 1.71 & 2.49 & 0.6 & 0.14 & 0. & 497 & 65 & 14 & 100 & 35 & 33 & 54 & 57 & 14 & 164 & 160 & 25 \\
\hline $7-3,30.0-35.0$ & 56.92 & 56.54 & 14.43 & 4.5 & 3.09 & 5.97 & 1.93 & 2.73 & 0.60 & 0.13 & 0.1 & 494 & 63 & 13 & 101 & 39 & 32 & 60 & 55 & 13 & 195 & 153 & 25 \\
\hline $8-2,60.0-65.0$ & 65.53 & 52.54 & 14.12 & 5.06 & 3.00 & 5.89 & 1.63 & 2.42 & 0.58 & 0.13 & 0.1 & 465 & 65 & 15 & 79 & 38 & 31 & 51 & 59 & 13 & 177 & 146 & 24 \\
\hline $9-4,53.0-58.0$ & 78.05 & 57.74 & 16.43 & 5.74 & 2.93 & 3.30 & 1.78 & 2.81 & 0.6 & 0.14 & 0.1 & 538 & 67 & 14 & 100 & 37 & 36 & 57 & 56 & 16 & 149 & 158 & 26 \\
\hline $10-2,71.0-76.0$ & 84.84 & 54.27 & 16.22 & 5.94 & 3.27 & 4.45 & 1.69 & 2.88 & 0.64 & 0.14 & 0.10 & 493 & 65 & 14 & 114 & 37 & 35 & 63 & 58 & 15 & 149 & 173 & 26 \\
\hline $11-3,49$ & & 55.63 & 15.34 & 5.5 & 2.90 & 6.50 & 1.57 & 2.45 & 0.59 & 0.15 & 0.20 & 438 & 65 & 16 & 98 & 39 & 32 & 59 & 63 & 14 & 193 & 189 & 26 \\
\hline $16-3,50.0-55.0$ & 145.92 & 60.65 & 15.84 & 5.56 & 3.29 & 4.45 & 1.64 & 2.91 & 0.62 & 0.12 & 0.11 & 498 & 63 & 14 & 103 & 36 & 35 & 63 & 53 & 15 & 149 & 167 & 25 \\
\hline $17-2,34.0-37.0$ & 153.86 & 51.66 & 11.56 & 3.85 & 2.91 & 4.93 & 1.63 & 2.25 & 0.50 & 0.08 & 0.09 & 522 & 56 & 12 & 104 & 30 & 28 & 46 & 46 & 10 & 171 & 105 & 22 \\
\hline $18-1,10.0-14.0$ & 162.32 & 54.81 & 11.15 & 3.73 & 3.21 & 5.23 & 1.81 & 2.29 & 0.50 & 0.08 & 0.09 & 539 & 55 & 12 & 78 & 29 & 27 & 35 & 46 & 9 & 172 & 92 & 22 \\
\hline $20, \mathrm{CC}(24.0-25.0)$ & 181.29 & 66.12 & 9.21 & 2.73 & 2.79 & 5.33 & 1.92 & 2.21 & 0.39 & 0.05 & 0.06 & 600 & 50 & 9 & 55 & 25 & 24 & 23 & 33 & 6 & 201 & 54 & 21 \\
\hline $21-2,46.0-49.0$ & 192.18 & 58.01 & 14.27 & 5.9 & 3.08 & 5.51 & 1.67 & 2.75 & 0.5 & 0.1 & 0. & 488 & 62 & 15 & 96 & 36 & 33 & 58 & 63 & 13 & 185 & 161 & 26 \\
\hline $22-1,40.0-45.0$ & 209.72 & 84.93 & 7.33 & 1.92 & 1.52 & 3.55 & 1.98 & 1.83 & 0.35 & 0.01 & 0. & 612 & 46 & 7 & 46 & 15 & 25 & 13 & 24 & 4 & 213 & 36 & 15 \\
\hline $24-2,35.0-38.0$ & 249.57 & 74.78 & 8.51 & 2.19 & 2.30 & 4.81 & 1.99 & 2.07 & 0.32 & 0.04 & 0. & 612 & 44 & 8 & 68 & 22 & 24 & 19 & 32 & 5 & 223 & 50 & 19 \\
\hline $28-2,12.0-18.0$ & 306.95 & 58.01 & 14.7 & 5. & 3.7 & 6.35 & 1.37 & 2.90 & 0.6 & 0.11 & 0.1 & 454 & 63 & 14 & 110 & 37 & 32 & 60 & 58 & 14 & 159 & 147 & 25 \\
\hline $30-6,35.0-38.0$ & 332.16 & 51.01 & 15.99 & 5.79 & 3.49 & 5.49 & 1.43 & 2.98 & 0.65 & 0.11 & 0.10 & 471 & 66 & 15 & 118 & 39 & 35 & 64 & 58 & 15 & 162 & 167 & 26 \\
\hline $32-4,110.0-113.0$ & 348.71 & 55.26 & 16.03 & 5.0 & 3.5 & 5.31 & 1.64 & 3.37 & 0.6 & 0. & 0. & 478 & 67 & 15 & 101 & 45 & 35 & 70 & 64 & 15 & 169 & 145 & 26 \\
\hline $33-2,106.0-107.0$ & 354.10 & 46.07 & 14.85 & 5.25 & 3.25 & 5.92 & 1.39 & 2.85 & 0.64 & 0.14 & 0.17 & 461 & 67 & 14 & 83 & 37 & 32 & 58 & 58 & 14 & 177 & 148 & 25 \\
\hline $34-3,43.0-46.0$ & 365.15 & 56.63 & 16.50 & 5.70 & 3.17 & 5.34 & 1.46 & 3.15 & 0.66 & 0.15 & 0.17 & 456 & 72 & 15 & 110 & 42 & 36 & 104 & 60 & 16 & 177 & 172 & 26 \\
\hline
\end{tabular}




\begin{tabular}{|c|c|c|c|c|c|c|c|c|c|c|c|c|c|c|c|c|c|c|c|c|c|c|c|}
\hline \multirow{2}{*}{$\begin{array}{c}\text { Core-Section } \\
\text { (interval in cm) }\end{array}$} & \multirow{2}{*}{$\begin{array}{l}\text { Sub-bottom } \\
\text { depth (m) }\end{array}$} & \multicolumn{10}{|c|}{ Major elements (wt.\%) } & \multicolumn{12}{|c|}{ Trace elements (ppm) } \\
\hline & & $\mathrm{SiO}_{2}$ & $\mathrm{Al}_{2} \mathrm{O}_{3}$ & $\mathrm{FeO} / \mathrm{Fe}_{2} \mathrm{O}_{3}$ & $\mathrm{MgO}$ & $\mathrm{CaO}$ & $\mathrm{Na}_{2} \mathrm{O}$ & $\mathrm{K}_{2} \mathrm{O}$ & $\mathrm{TiO}_{2}$ & $\mathrm{P}_{2} \mathrm{O}_{5}$ & $\mathrm{MnO}$ & $\mathrm{Ba}$ & $\mathrm{Ce}$ & Co & $\mathrm{Cr}$ & $\mathrm{Cu}$ & $\mathrm{La}$ & $\mathrm{Li}$ & $\mathrm{Ni}$ & $\mathrm{Sc}$ & $\mathrm{Sr}$ & V & $\mathrm{Y}$ \\
\hline \multicolumn{24}{|l|}{ Hole 620} \\
\hline $13-2,60.0-65.0$ & 109.35 & 53.60 & 15.53 & 5.40 & 3.13 & 4.54 & 2.38 & 2.83 & 0.64 & 0.14 & 0.13 & 519 & 68 & 14 & 94 & 37 & 37 & 57 & 52 & 15 & 178 & 149 & 27 \\
\hline $14-2,60.0-65.0$ & 119.13 & 53.97 & 15.71 & 5.39 & 3.30 & 4.45 & 2.20 & 2.95 & 0.63 & 0.14 & 0.11 & 528 & 68 & 14 & 101 & 38 & 37 & 57 & 52 & 15 & 171 & 146 & 26 \\
\hline $15-2,60.0-65.0$ & 128.73 & 56.04 & 15.12 & 5.26 & 2.90 & 4.08 & 1.72 & 2.66 & 0.62 & 0.13 & 0.12 & 502 & 70 & 14 & 100 & 31 & 35 & 51 & 51 & 15 & 156 & 138 & 26 \\
\hline $16-2,60.0-65.0$ & 138.33 & 52.86 & 14.46 & 5.60 & 2.86 & 5.34 & 1.90 & 2.44 & 0.59 & 0.14 & 0.19 & 441 & 69 & 15 & 109 & 34 & 34 & 53 & 57 & 14 & 176 & 165 & 26 \\
\hline $17-2,60.0-65.0$ & 147.92 & 54.34 & 15.55 & 5.65 & 2.91 & 3.54 & 2.02 & 2.59 & 0.61 & 0.13 & 0.11 & 477 & 68 & 14 & 101 & 33 & 35 & 53 & 52 & 15 & 143 & 151 & 26 \\
\hline $17-6,60.0-65.0$ & 153.92 & 54.78 & 15.81 & 5.67 & 2.92 & 3.50 & 2.07 & 2.63 & 0.62 & 0.13 & 0.10 & 494 & 67 & 14 & 87 & 33 & 36 & 53 & 51 & 15 & 144 & 144 & 26 \\
\hline $18-2,60.0-65.0$ & 157.52 & 55.94 & 15.54 & 5.47 & 2.98 & 4.16 & 2.11 & 2.74 & 0.63 & 0.14 & 0.15 & 504 & 67 & 14 & 99 & 35 & 36 & 55 & 53 & 15 & 165 & 161 & 27 \\
\hline $19-1,60.0-65.0$ & 165.63 & 56.33 & 14.93 & 4.92 & 3.29 & 4.74 & 1.72 & 2.90 & 0.61 & 0.13 & 0.11 & 495 & 66 & 13 & 101 & 32 & 36 & 53 & 54 & 15 & 159 & 148 & 25 \\
\hline $20-4,60.0-65.0$ & 179.73 & 55.93 & 14.90 & 5.43 & 2.92 & 4.60 & 1.70 & 2.50 & 0.62 & 0.15 & 0.12 & 485 & 68 & 14 & 103 & 32 & 35 & 47 & 55 & 15 & 162 & 165 & 26 \\
\hline $21-2,60.0-65.0$ & 186.13 & 54.53 & 15.51 & 5.85 & 3.22 & 4.16 & 1.70 & 2.79 & 0.64 & 0.15 & 0.12 & 475 & 69 & 14 & 103 & 33 & 36 & 51 & 59 & 16 & 151 & 156 & 27 \\
\hline $22-2,60.0-65.0$ & 195.52 & 56.27 & 14.80 & 5.38 & 2.88 & 4.30 & 1.82 & 2.49 & 0.60 & 0.15 & 0.18 & 493 & 66 & 13 & 102 & 34 & 35 & 50 & 56 & 15 & 167 & 167 & 26 \\
\hline $23-2,59.0-63.0$ & 204.91 & 57.42 & 14.80 & 5.49 & 3.21 & 4.78 & 1.77 & 2.75 & 0.61 & 0.14 & 0.18 & 475 & 67 & 14 & 106 & 33 & 35 & 51 & 61 & 15 & 165 & 152 & 25 \\
\hline $24-1,60.0-65.0$ & 212.92 & 57.99 & 14.20 & 5.55 & 3.15 & 5.02 & 1.65 & 2.59 & 0.62 & 0.14 & 0.14 & 470 & 67 & 15 & 107 & 32 & 34 & 47 & 58 & 15 & 164 & 161 & 25 \\
\hline $25-1,50.0-52.0$ & 222.31 & 57.85 & 12.38 & 4.65 & 3.61 & 6.33 & 1.61 & 2.55 & 0.57 & 0.12 & 0.11 & 476 & 62 & 15 & 94 & 31 & 30 & 41 & 52 & 13 & 165 & 123 & 23 \\
\hline $26-2,60.0-65.0$ & 233.42 & 55.20 & 12.93 & 4.80 & 3.49 & 5.90 & 1.67 & 2.61 & 0.59 & 0.12 & 0.11 & 490 & 64 & 15 & 94 & 30 & 31 & 43 & 52 & 13 & 166 & 130 & 23 \\
\hline $27-1,60.0-65.0$ & 241.52 & 54.33 & 14.56 & 5.52 & 2.99 & 4.89 & 1.52 & 2.61 & 0.60 & 0.14 & 0.13 & 483 & 67 & 15 & 84 & 33 & 34 & 49 & 57 & 15 & 165 & 154 & 25 \\
\hline $28-2,45.0-50.0$ & 252.48 & 57.84 & 13.23 & 4.87 & 3.32 & 5.95 & 1.68 & 2.63 & 0.59 & 0.13 & 0.12 & 483 & 66 & 14 & 83 & 30 & 32 & 46 & 52 & 14 & 175 & 138 & 24 \\
\hline $29-1,60.0-65.0$ & 260.72 & 59.32 & 11.99 & 4.25 & 3.54 & 5.34 & 1.67 & 2.62 & 0.54 & 0.10 & 0.08 & 520 & 63 & 13 & 90 & 28 & 32 & 43 & 46 & 12 & 166 & 97 & 22 \\
\hline $30-2,60.0-65.0$ & 271.83 & 53.92 & 14.42 & 5.20 & 3.35 & 5.31 & 1.53 & 2.88 & 0.61 & 0.12 & 0.10 & 480 & 68 & 15 & 86 & 31 & 35 & 56 & 54 & 15 & 161 & 130 & 26 \\
\hline $31-2,60.0-65.0$ & 281.42 & 53.43 & 15.24 & 5.62 & 3.39 & 4.95 & 1.74 & 3.02 & 0.62 & 0.13 & 0.10 & 486 & 71 & 16 & 94 & 33 & 36 & 59 & 55 & 16 & 158 & 140 & 26 \\
\hline $32-2,60.0-65.0$ & 291.03 & 53.42 & 14.06 & 5.12 & 3.45 & 5.39 & 1.79 & 2.79 & 0.61 & 0.12 & 0.10 & 499 & 68 & 15 & 107 & 32 & 35 & 53 & 54 & 14 & 162 & 134 & 24 \\
\hline $33-2,60.0-65.0$ & 300.63 & 52.42 & 14.89 & 5.47 & 3.73 & 6.02 & 1.70 & 3.05 & 0.63 & 0.13 & 0.10 & 449 & 72 & 16 & 106 & 32 & 36 & 61 & 58 & 16 & 165 & 144 & 26 \\
\hline $33-5,60.0-65.0$ & 305.13 & 51.92 & 14.57 & 5.42 & 3.63 & 5.88 & 1.59 & 2.96 & 0.63 & 0.12 & 0.12 & 445 & 70 & 15 & 104 & 33 & 35 & 63 & 56 & 14 & 162 & 141 & 26 \\
\hline $35-2,60.0-65.0$ & 319.83 & 52.41 & 14.88 & 5.41 & 3.45 & 5.48 & 1.86 & 3.05 & 0.63 & 0.13 & 0.18 & 468 & 68 & 15 & 101 & 37 & 35 & 66 & 57 & 14 & 174 & 150 & 27 \\
\hline $36-2,60.0-65.0$ & 329.42 & 54.02 & 14.98 & 5.49 & 3.41 & 5.26 & 1.80 & 3.01 & 0.63 & 0.13 & 0.21 & 479 & 69 & 16 & 101 & 38 & 36 & 69 & 58 & 14 & 172 & 150 & 27 \\
\hline $37-2,60.0-65.0$ & 339.03 & 53.39 & 13.40 & 4.69 & 3.72 & 6.26 & 1.79 & 2.83 & 0.58 & 0.11 & 0.21 & 467 & 67 & 15 & 88 & 35 & 33 & 57 & 53 & 12 & 179 & 124 & 25 \\
\hline $38-2,60.0-65.0$ & 348.63 & 52.25 & 15.18 & 5.52 & 3.66 & 5.46 & 1.81 & 3.15 & 0.63 & 0.13 & 0.17 & 459 & 70 & 16 & 84 & 37 & 36 & 67 & 57 & 15 & 166 & 144 & 27 \\
\hline $40-2,60.0-65.0$ & 367.63 & 50.41 & 14.06 & 5.47 & 3.93 & 6.68 & 1.57 & 2.90 & 0.64 & 0.12 & 0.09 & 425 & 74 & 16 & 106 & 33 & 34 & 58 & 57 & 14 & 153 & 127 & 26 \\
\hline $41-4,60.0-65.0$ & 380.13 & 48.00 & 13.65 & 5.14 & 4.93 & 8.17 & 1.81 & 3.20 & 0.61 & 0.13 & 0.08 & 412 & 71 & 16 & 102 & 37 & 33 & 62 & 55 & 13 & 170 & 113 & 27 \\
\hline $42-2,60.0-65.0$ & 386.63 & 52.24 & 13.75 & 5.82 & 4.49 & 5.96 & 1.75 & 2.99 & 0.63 & 0.15 & 0.10 & 418 & 74 & 16 & 102 & 33 & 34 & 53 & 55 & 13 & 139 & 117 & 28 \\
\hline $43-1,35.0-40.0$ & 394.38 & 49.59 & 13.82 & 5.50 & 4.40 & 8.21 & 1.77 & 3.17 & 0.63 & 0.14 & 0.09 & 417 & 75 & 17 & 97 & 36 & 34 & 58 & 56 & 13 & 180 & 115 & 27 \\
\hline $44-3,60.0-65.0$ & 407.13 & 52.16 & 14.66 & 5.86 & 3.95 & 5.39 & 2.04 & 3.05 & 0.67 & 0.15 & 0.11 & 438 & 77 & 16 & 102 & 35 & 37 & 56 & 55 & 14 & 153 & 126 & 29 \\
\hline \multicolumn{24}{|l|}{ Hole 621} \\
\hline $1-2,60.0-65.0$ & 2.13 & 9.32 & 15.08 & 6.57 & 2.57 & 2.45 & 2.06 & 2.72 & 0.65 & 0.12 & 0.1 & 534 & 70 & 16 & 93 & 29 & 38 & 48 & 50 & 14 & 139 & 143 & 27 \\
\hline $2-3,60.0-65.0$ & 7.13 & 59.59 & 15.01 & 5.34 & 2.50 & 2.57 & 1.92 & 2.66 & 0.64 & 0.13 & 0.10 & 540 & 71 & 16 & 78 & 28 & 38 & 46 & 51 & 14 & 143 & 142 & 27 \\
\hline $3-3,60.0-65.0$ & 16.73 & 62.21 & 14.49 & 5.17 & 2.48 & 2.57 & 1.90 & 2.63 & 0.66 & 0.13 & 0.10 & 579 & 70 & 16 & 79 & 28 & 38 & 45 & 48 & 13 & 153 & 134 & 28 \\
\hline $4-3,60.0-65.0$ & 26.33 & 61.50 & 15.33 & 5.60 & 2.42 & 2.22 & 1.80 & 2.59 & 0.67 & 0.14 & 0.11 & 568 & 73 & 17 & 93 & 29 & 39 & 48 & 50 & 14 & 148 & 146 & 28 \\
\hline $5-3,60.0-65.0$ & 35.93 & 59.10 & 15.63 & 5.52 & 2.49 & 2.29 & 1.95 & 2.82 & 0.67 & 0.13 & 0.11 & 580 & 71 & 16 & 88 & 30 & 39 & 51 & 48 & 14 & 153 & 138 & 28 \\
\hline $6-3,45.0-50.0$ & 45.38 & 7.48 & 15.59 & 5.57 & 2.76 & 2. & 1.85 & 2.84 & 0.66 & 0.14 & 0.10 & 543 & 74 & 17 & 93 & 32 & 40 & 133 & 51 & 15 & 149 & 156 & 28 \\
\hline $7-3,60.0-65.0$ & 49.82 & 63.94 & 15.01 & 5.54 & 2.64 & 2.53 & 1.66 & 2.59 & 0.64 & 0.14 & 0.10 & 511 & 74 & 17 & 103 & 32 & 38 & 48 & 52 & 15 & 138 & 160 & 27 \\
\hline $8-2,60.0-65.0$ & 57.82 & 58.17 & 14.71 & 5. & 2.64 & 2.50 & 1.67 & 2.53 & 0.64 & 0.14 & 0.11 & 512 & 74 & 17 & 97 & 29 & 38 & 47 & 52 & 14 & 141 & 157 & 28 \\
\hline $9-2,60.0-65.0$ & 62.32 & 64.10 & 16.63 & 6.05 & 3.00 & 2.97 & 1.84 & 2.85 & 0.68 & 0.16 & 0.12 & 585 & 85 & 20 & 95 & 33 & 42 & 53 & 59 & 16 & 161 & 166 & 31 \\
\hline $10-2,60.0-65.0$ & 67.32 & 61.64 & 14.75 & 5.30 & 2.73 & 2.56 & 1.83 & 2.77 & 0.63 & 0.14 & 0.11 & 562 & 74 & 17 & 89 & 30 & 39 & 52 & 51 & 14 & 149 & 150 & 28 \\
\hline $11-2,60.0-65.0$ & 72.52 & 65.24 & 14.08 & 5.23 & 2.65 & 2.71 & 1.81 & 2.59 & 0.61 & 0.13 & 0.11 & 530 & 74 & 17 & 90 & 29 & 38 & 48 & 52 & 14 & 145 & 148 & 27 \\
\hline $12-2,60.0-65.0$ & 80.93 & 61.24 & 14.21 & 5.05 & 2.68 & 2.54 & 1.86 & 2.77 & 0.62 & 0.13 & 0.11 & 571 & 72 & 17 & 91 & 31 & 38 & 53 & 51 & 13 & 150 & 142 & 27 \\
\hline $13-2,60.0-65.0$ & 83.52 & 67.18 & 14.30 & 5.10 & 2.71 & 2.57 & 1.93 & 2.78 & 0.60 & 0.13 & 0.1 & 583 & 70 & 16 & 87 & 29 & 37 & 55 & 50 & 14 & 148 & 143 & 27 \\
\hline $14-2,60.0-65.0$ & 88.82 & 62.00 & 14.61 & 5.12 & 2.71 & 2.42 & 2.01 & 2.85 & 0.62 & 0.13 & 0.10 & 569 & 70 & 17 & 63 & 31 & 37 & 54 & 50 & 14 & 148 & 144 & 27 \\
\hline $15-2,60.0-65.0$ & 96.32 & 60.72 & 14.19 & 5.23 & 2.66 & 2.92 & 1.66 & 2.48 & 0.63 & 0.13 & 0.11 & 550 & 75 & 17 & 90 & 28 & 38 & 46 & 52 & 13 & 150 & 144 & 28 \\
\hline $16-2,60.0-65.0$ & 101.82 & 62.0 & 13.7 & 5.1 & 2.6 & 2.8 & 1. & 2.50 & 0.6 & 0.1 & 0.11 & 538 & 74 & 17 & 89 & 29 & 37 & 43 & 52 & 13 & 149 & 140 & 27 \\
\hline $17-2,60.0-65.0$ & 106.82 & 58.38 & 13.99 & 5.07 & 2.66 & 2.75 & 1.77 & 2.62 & 0.61 & 0.13 & 0.11 & 553 & 70 & 17 & 69 & 28 & 37 & 48 & 50 & 13 & 153 & 140 & 27 \\
\hline $19-2,60.0-65.0$ & 125.82 & 59.37 & 13.61 & 4.98 & 2.47 & 2.62 & 1.73 & 2.56 & 0.58 & 0.11 & 0.1 & 540 & 68 & 17 & 73 & 27 & 36 & 44 & 52 & 12 & 157 & 126 & 25 \\
\hline $20-2,60.0-65.0$ & 132.33 & 56.74 & 13.93 & 5.12 & 2.58 & 2.70 & 1.72 & 2.54 & 0.60 & 0.12 & 0.11 & 531 & 71 & 17 & 96 & 28 & 36 & 46 & 52 & 13 & 153 & 134 & 27 \\
\hline
\end{tabular}




\begin{tabular}{|c|c|c|c|c|c|c|c|c|c|c|c|c|c|c|c|c|c|c|c|c|c|c|c|}
\hline \multirow{2}{*}{$\begin{array}{l}\text { Core-Section } \\
\text { (interval in cm) }\end{array}$} & \multirow{2}{*}{$\begin{array}{l}\text { Sub-bottom } \\
\text { depth (m) }\end{array}$} & \multicolumn{10}{|c|}{ Major elements (wt.\%) } & \multicolumn{12}{|c|}{ Trace elements (ppm) } \\
\hline & & $\mathrm{SiO}_{2}$ & $\mathrm{Al}_{2} \mathrm{O}_{3}$ & $\mathrm{FeO} / \mathrm{Fe}_{2} \mathrm{O}_{3}$ & $\mathrm{MgO}$ & $\mathrm{CaO}$ & $\mathrm{Na}_{2} \mathrm{O}$ & $\mathrm{K}_{2} \mathrm{O}$ & $\mathrm{TiO}_{2}$ & $\mathrm{P}_{2} \mathrm{O}_{5}$ & $\mathrm{MnO}$ & $\mathrm{Ba}$ & $\mathrm{Ce}$ & Co & $\mathrm{Cr}$ & $\mathrm{Cu}$ & $\mathrm{La}$ & $\mathrm{Li}$ & $\mathrm{Ni}$ & Sc & $\mathrm{Sr}$ & V & $\mathrm{Y}$ \\
\hline \multicolumn{24}{|l|}{ Hole 621 (Cont.) } \\
\hline $22-2,60.0-65.0$ & 135.63 & 67.17 & 12.94 & 4.68 & 2.36 & 2.66 & 1.73 & 2.47 & 0.55 & 0.10 & 0.10 & 546 & 65 & 16 & 92 & 26 & 34 & 41 & 48 & 12 & 162 & 119 & 24 \\
\hline $23-2,60.0-65.0$ & 139.42 & 61.79 & 10.07 & 4.60 & 2.23 & 2.37 & 1.74 & 2.48 & 0.53 & 0.08 & 0.09 & 503 & 56 & 14 & 53 & 28 & 28 & 42 & 45 & 8 & 154 & 115 & 20 \\
\hline $24-2,60.0-65.0$ & 149.23 & 59.17 & 14.50 & 5.28 & 2.73 & 2.77 & 1.79 & 2.79 & 0.65 & 0.13 & 0.11 & 512 & 73 & 17 & 83 & 33 & 38 & 52 & 51 & 13 & 152 & 135 & 27 \\
\hline $25, \mathrm{CC}(18.0-20.0)$ & 159.51 & 59.88 & 14.63 & 5.04 & 2.68 & 2.89 & 1.86 & 2.85 & 0.67 & 0.11 & 0.11 & 525 & 74 & 17 & 80 & 32 & 38 & 54 & 52 & 13 & 148 & 130 & 27 \\
\hline $26-2,60.0-65.0$ & 168.42 & 60.29 & 12.65 & 4.55 & 2.45 & 2.77 & 1.77 & 2.57 & 0.56 & 0.10 & 0.10 & 543 & 66 & 14 & 69 & 27 & 34 & 42 & 46 & 11 & 162 & 108 & 23 \\
\hline $27-2,45.0-50.0$ & 177.88 & 79.27 & 8.51 & 2.28 & 1.78 & 3.03 & 1.95 & 2.14 & 0.41 & 0.04 & 0.04 & 559 & 53 & 10 & 52 & 24 & 27 & 54 & 31 & 6 & 192 & 51 & 19 \\
\hline $29-3,60.0-65.0$ & 198.73 & 66.56 & 10.86 & 3.77 & 2.42 & 3.55 & 1.74 & 2.29 & 0.48 & 0.07 & 0.09 & 539 & 60 & 14 & 81 & 25 & 30 & 37 & 45 & 9 & 168 & 104 & 20 \\
\hline $31-1,66.0-70.0$ & 200.88 & 58.08 & 13.37 & 4.61 & 3.09 & 4.77 & 1.63 & 2.94 & 0.67 & 0.09 & 0.10 & 1993 & 69 & 17 & 83 & 32 & 33 & 53 & 55 & 13 & 176 & 123 & 24 \\
\hline \multicolumn{24}{|l|}{ Hole 623} \\
\hline $1-1,90.0-95.0$ & 0.92 & 53.94 & 17.33 & 5.62 & 3.29 & 2.40 & 3.15 & 3.52 & 0.71 & 0.18 & 0.11 & 481 & 60 & 13 & 107 & 42 & 42 & 69 & 37 & 16 & 144 & 164 & 28 \\
\hline $1-4,90.0-95.0$ & 5.43 & 57.58 & 16.12 & 5.35 & 3.22 & 3.01 & 2.69 & 3.37 & 0.66 & 0.16 & 0.13 & 486 & 46 & 13 & 118 & 40 & 37 & 64 & 38 & 14 & 144 & 147 & 26 \\
\hline $2-3,60.0-65.0$ & 10.23 & 55.58 & 15.94 & 6.03 & 3.04 & 3.41 & 2.24 & 2.57 & 0.69 & 0.19 & 0.17 & 514 & 48 & 14 & 112 & 36 & 34 & 57 & 40 & 16 & 146 & 184 & 26 \\
\hline $2-6,60.0-65.0$ & 14.73 & 55.49 & 15.38 & 5.81 & 3.03 & 3.85 & 2.07 & 2.43 & 0.65 & 0.16 & 0.11 & 493 & 37 & 14 & 125 & 35 & 34 & 58 & 39 & 15 & 142 & 203 & 25 \\
\hline $3-2,60.0-65.0$ & 18.33 & 54.29 & 14.51 & 6.05 & 3.13 & 4.34 & 1.84 & 2.29 & 0.66 & 0.17 & 0.11 & 475 & 40 & 13 & 145 & 32 & 34 & 51 & 40 & 15 & 142 & 209 & 25 \\
\hline $3-5,60.0-65.0$ & 22.83 & 54.89 & 16.68 & 5.93 & 3.01 & 2.60 & 2.13 & 2.85 & 0.67 & 0.17 & 0.11 & 506 & 34 & 14 & 106 & 36 & 30 & 57 & 38 & 16 & 136 & 172 & 25 \\
\hline $4-3,60.0-65.0$ & 29.42 & 56.28 & 16.56 & 5.81 & 3.00 & 3.10 & 2.18 & 2.80 & 0.66 & 0.16 & 0.11 & 527 & 30 & 12 & 114 & 35 & 30 & 60 & 33 & 15 & 137 & 169 & 23 \\
\hline $5-1,60.0-65.0$ & 36.03 & 56.01 & 14.35 & 5.68 & 2.90 & 3.88 & 1.77 & 2.29 & 0.63 & 0.15 & 0.11 & 499 & 32 & 13 & 126 & 30 & 30 & 49 & 33 & 14 & 134 & 177 & 23 \\
\hline $5-4,60.0-65.0$ & 40.53 & 56.75 & 15.56 & 5.54 & 2.89 & 3.41 & 2.02 & 2.66 & 0.65 & 0.15 & 0.11 & 532 & 29 & 12 & 96 & 35 & 32 & 58 & 33 & 14 & 146 & 176 & 23 \\
\hline $6-2,60.0-65.0$ & 47.13 & 56.04 & 15.11 & 5.24 & 2.97 & 3.61 & 2.00 & 2.67 & 0.64 & 0.16 & 0.14 & 499 & 29 & 13 & 99 & 32 & 29 & 58 & 35 & 14 & 149 & 178 & 22 \\
\hline $6-5,60.0-65.0$ & 51.63 & 56.53 & 15.17 & 5.58 & 3.14 & 4.62 & 1.97 & 2.63 & 0.64 & 0.16 & 0.13 & 511 & 30 & 14 & 93 & 35 & 29 & 58 & 38 & 14 & 155 & 172 & 22 \\
\hline $7-3,60.0-65.0$ & 58.22 & 68.46 & 9.52 & 2.78 & 2.06 & 3.56 & 1.93 & 2.07 & 0.42 & 0.09 & 0.07 & 505 & 0 & 5 & 40 & 18 & 17 & 26 & 15 & 6 & 175 & 73 & 15 \\
\hline $8-2,95.0-100.0$ & 66.68 & 56.12 & 14.60 & 4.78 & 3.29 & 4.27 & 2.20 & 2.80 & 0.61 & 0.15 & 0.12 & 490 & 24 & 12 & 90 & 33 & 28 & 53 & 31 & 12 & 159 & 143 & 21 \\
\hline $9-2,60.0-65.0$ & 75.93 & 57.20 & 11.79 & 4.07 & 3.40 & 5.80 & 2.11 & 2.35 & 0.55 & 0.14 & 0.10 & 575 & 15 & 11 & 87 & 25 & 24 & 51 & 23 & 9 & 170 & 103 & 19 \\
\hline $10-2,25.0-30.0$ & 85.17 & 60.72 & 9.58 & 3.20 & 3.23 & 5.99 & 1.86 & 1.94 & 0.51 & 0.13 & 0.09 & 572 & 15 & 8 & 71 & 18 & 21 & 27 & 19 & 7 & 188 & 82 & 19 \\
\hline $11-2,50.0-65.0$ & 95.13 & 57.06 & 10.61 & 3.92 & 3.42 & 6.57 & 1.75 & 2.02 & 0.58 & 0.14 & 0.10 & 577 & 20 & 10 & 79 & 24 & 25 & 33 & 21 & 9 & 171 & 102 & 21 \\
\hline $12-2,60.0-65.0$ & 104.73 & 54.42 & 15.80 & 5.33 & 3.02 & 3.70 & 1.92 & 2.72 & 0.64 & 0.16 & 0.17 & 454 & 30 & 13 & 101 & 35 & 29 & 59 & 38 & 14 & 148 & 185 & 22 \\
\hline $12-4,60.0-65.0$ & 107.73 & 56.31 & 15.79 & 5.09 & 2.89 & 3.56 & 1.79 & 2.82 & 0.63 & 0.15 & 0.13 & 524 & 28 & 12 & 109 & 34 & 32 & 58 & 33 & 14 & 146 & 177 & 23 \\
\hline $14-1,59.0-64.0$ & 122.01 & 54.23 & 15.62 & 5.73 & 2.92 & 5.54 & 1.75 & 2.61 & 0.63 & 0.17 & 0.19 & 467 & 40 & 16 & 119 & 36 & 31 & 60 & 44 & 14 & 175 & 190 & 24 \\
\hline $15-1,60.0-65.0$ & 131.42 & 79.51 & 6.29 & 1.87 & 1.13 & 2.68 & 1.80 & 1.63 & 0.30 & 0.05 & 0.06 & 495 & 0 & 1 & 0 & 7 & 12 & 11 & 1 & 2 & 174 & 26 & 11 \\
\hline $16-2,60.0-65.0$ & 142.23 & 70.53 & 7.89 & 2.23 & 2.01 & 4.54 & 1.84 & 1.95 & 0.34 & 0.09 & 0.07 & 574 & 0 & 4 & 28 & 12 & 18 & 17 & 7 & 3 & 189 & 40 & 19 \\
\hline $17-1,60.0-65.0$ & 150.02 & 56.18 & 15.48 & 5.38 & 3.02 & 4.33 & 1.80 & 2.79 & 0.64 & 0.16 & 0.14 & 480 & 34 & 13 & 101 & 33 & 32 & 59 & 33 & 14 & 155 & 168 & 23 \\
\hline \multicolumn{24}{|l|}{ Hole 618} \\
\hline $4-2,86.0-90.0$ & 28.08 & 48.15 & 13.07 & 4.58 & 3.07 & 8.17 & 3.58 & 2.76 & 0.53 & 0.14 & 0.1 & 447 & 28 & 13 & 107 & 34 & 28 & 55 & 32 & 11 & 295 & 114 & 20 \\
\hline $5-2,81.0-85.0$ & 37.63 & 52.29 & 12.86 & 4.32 & 3.57 & 7.41 & 2.53 & 2.78 & 0.58 & 0.14 & 0.0 & 432 & 38 & 13 & 104 & 30 & 29 & 51 & 33 & 11 & 201 & 100 & 20 \\
\hline $6-2,41.0-46.0$ & 46.83 & 52.14 & 12.65 & 4.95 & 3.47 & 7.33 & 2.36 & 2.84 & 0.56 & 0.13 & 0.09 & 426 & 33 & 13 & 107 & 28 & 28 & 50 & 33 & 11 & 204 & 99 & 19 \\
\hline $7-1,70.0-75.0$ & 51.63 & 54.16 & 13.37 & 4.51 & 3.53 & 8.43 & 2.54 & 2.87 & 0.57 & 0.14 & 0.09 & 447 & 36 & 13 & 94 & 31 & 29 & 53 & 34 & 11 & 206 & 99 & 20 \\
\hline $8-3,90.0-95.0$ & 60.83 & 51.10 & 12.13 & 4.53 & 3.37 & 8.34 & 2.19 & 2.58 & 0.57 & 0.13 & 0.09 & 441 & 35 & 13 & 107 & 29 & 31 & 48 & 44 & 11 & 206 & 100 & 21 \\
\hline $9-1,80.0-85.0$ & 70.72 & 52.14 & 12.67 & 4.71 & 3.57 & 8.29 & 2.19 & 2.74 & 0.59 & 0.14 & 0.10 & 442 & 41 & 13 & 86 & 26 & 31 & 48 & 34 & 11 & 195 & 106 & 22 \\
\hline $10-1,85.0-90.0$ & 77.78 & 50.60 & 11.99 & 4.69 & 3.37 & 8.52 & 2.08 & 2.47 & 0.56 & 0.14 & 0.10 & 436 & 38 & 13 & 104 & 30 & 27 & 44 & 35 & 11 & 191 & 104 & 21 \\
\hline \multicolumn{24}{|l|}{ Hole 619} \\
\hline $1-1,50.0-52.0$ & 1.51 & 47.25 & 14.25 & 4.58 & 3.51 & 7.32 & 2.78 & 3.15 & 0.5 & 0.15 & 0.1 & 453 & 37 & 14 & 106 & 61 & 29 & 58 & 37 & 12 & 255 & 127 & 21 \\
\hline $5-3,116.0-118.0$ & 34.07 & 52.18 & 13.70 & 5.55 & 2.79 & 6.98 & 1.66 & 2.43 & 0.62 & 0.16 & 0.2 & 441 & 44 & 16 & 119 & 31 & 30 & 53 & 41 & 13 & 194 & 190 & 25 \\
\hline $9-1,116.0-118.0$ & 69.87 & 44.33 & 13.77 & 4.98 & 4.51 & 8.60 & 1.74 & 3.27 & 0.65 & 0.14 & 0.1 & 410 & 44 & 16 & 113 & 30 & 30 & 63 & 38 & 13 & 164 & 119 & 22 \\
\hline $16-4,57.0-59.0$ & 141.08 & 50.97 & 16.33 & 5.51 & 2.73 & 6.66 & 1.93 & 3.14 & 0.62 & 0.16 & 0.18 & 465 & 54 & 14 & 106 & 34 & 38 & 73 & 44 & 14 & 300 & 139 & 23 \\
\hline $18-2,81.0-82.0$ & 157.71 & 56.93 & 16.46 & 5.75 & 2.58 & 2.01 & 1.55 & 3.03 & 0.79 & 0.17 & 0.09 & 415 & 61 & 12 & 86 & 27 & 41 & 70 & 30 & 15 & 142 & 140 & 28 \\
\hline $22-2,46.0-47.0$ & 189.26 & 59.48 & 15.00 & 4.66 & 2.56 & 3.27 & 1.80 & 3.20 & 0.68 & 0.14 & 0.08 & 435 & 41 & 11 & 96 & 28 & 35 & 62 & 30 & 12 & 171 & 111 & 24 \\
\hline
\end{tabular}

${ }^{a}$ Data from Mississippi Fan sites are plotted in Figure 2A-E. 
Table 2. X-ray clay diffraction data for Sites $614,615,616,617,620$, 621,622 , and 623 located on the Mississippi Fan.

\begin{tabular}{llllllllll}
\hline & & \multicolumn{4}{c}{ Clay fraction composition $(\%)$} \\
\cline { 4 - 8 } $\begin{array}{c}\text { Core-Section } \\
\text { (interval in } \mathrm{cm})\end{array}$ & $\begin{array}{c}\text { Sub-bottom } \\
\text { depth }(\mathrm{m})\end{array}$ & $\mathrm{Sm}$ & I & $\mathrm{Ch}$ & $\mathrm{K}$ & $\mathrm{Q}$ & $\mathrm{F}$ & $\mathrm{Ca}^{\mathrm{a}}$ & Do \\
\hline
\end{tabular}

Hole 614

$\begin{array}{lrrrrrrrrr}1-3,93-95 & 3.92 & 47 & 8 & 15 & 20 & 5 & 4 & 0 & 0 \\ 2-6,62-64 & 17.56 & 57 & 5 & 12 & 16 & 7 & 3 & 0 & 0 \\ 5-1,110-112 & 30.13 & 49 & 9 & 11 & 15 & 11 & 5 & 0 & 0 \\ 5-2,100-102 & 31.52 & 53 & 8 & 13 & 13 & 8 & 5 & 0 & 0 \\ 5-4,100-102 & 34.52 & 24 & 9 & 21 & 21 & 26 & 0 & 0 & 0\end{array}$

Hole $614 \mathrm{~A}$

$\begin{array}{rrrrrrrrrr}2-1,11-13 & 45.14 & 59 & 7 & 9 & 12 & 9 & 4 & 0 & 0 \\ 3-5,30-32 & 60.63 & 53 & 9 & 14 & 12 & 8 & 5 & 0 & 0 \\ 4-1,50-52 & 61.33 & 58 & 6 & 12 & 12 & 8 & 5 & 0 & 0 \\ 8-1,62-64 & 99.15 & 46 & 7 & 14 & 18 & 5 & 3 & 3 & 3 \\ 8-3,32-34 & 101.85 & 40 & 11 & 18 & 22 & 9 & 0 & 0 & 0 \\ 9-1,42-44 & 108.45 & 36 & 11 & 16 & 20 & 11 & 5 & 0 & 0 \\ 9-1,80-82 & 108.82 & 35 & 15 & 18 & 24 & 5 & 0 & 0 & 0 \\ 9-2,12-14 & 109.65 & 42 & 11 & 17 & 17 & 14 & 0 & 0 & 0\end{array}$

Hole $615^{\mathrm{a}}$

$\begin{array}{lrrrrrrrrr}1-1,60-62 & 0.63 & 25 & 14 & 18 & 28 & 9 & 6 & 0 & 0 \\ 3-2,55-57 & 14.17 & 47 & 8 & 14 & 18 & 10 & 3 & 0 & 0 \\ 5-4,62-64 & 38.14 & 60 & 7 & 10 & 12 & 8 & 2 & 0 & 0 \\ 6-4,85-87 & 47.88 & 48 & 9 & 15 & 15 & 7 & 7 & 0 & 0 \\ 6, C C(0-2) & 51.53 & 39 & 12 & 17 & 21 & 12 & 0 & 0 & 0 \\ 7-6,48-50 & 56.41 & 40 & 10 & 15 & 17 & 8 & 10 & 0 & 0 \\ 9-2,37-39 & 69.21 & 37 & 12 & 16 & 18 & 10 & 8 & 0 & 0 \\ 12-1,50-52 & 96.70 & 51 & 8 & 13 & 17 & 8 & 3 & 0 & 0 \\ 19-1,100-102 & 163.21 & 51 & 8 & 13 & 16 & 9 & 2 & 0 & 0 \\ 29-1,57-59 & 172.28 & 30 & 16 & 16 & 23 & 12 & 4 & 0 & 0 \\ 21-1,2-29 & 181.49 & 26 & 12 & 21 & 22 & 14 & 5 & 0 & 0 \\ 22-4,62-64 & 195.83 & 49 & 8 & 13 & 17 & 9 & 4 & 0 & 0 \\ 29-1,68-70 & 257.89 & 33 & 14 & 18 & 20 & 12 & 3 & 0 & 0 \\ 29-2,70-72 & 259.41 & 37 & 15 & 19 & 15 & 13 & 0 & 0 & 0 \\ 29-3,70-72 & 260.91 & 27 & 13 & 22 & 20 & 13 & 5 & 0 & 0 \\ 29-5,70-72 & 263.91 & 20 & 15 & 20 & 21 & 11 & 12 & 0 & 0 \\ 34-1,130-132 & 315.53 & 45 & 11 & 16 & 17 & 8 & 2 & 0 & 0 \\ 43-1,40-42 & 409.63 & 41 & 14 & 16 & 13 & 8 & 9 & 0 & 0 \\ 43-3,40-42 & 412.63 & 47 & 11 & 16 & 16 & 7 & 3 & 0 & 0 \\ 43-3,80-82 & 413.03 & 0 & 22 & 25 & 27 & 13 & 13 & 0 & 0 \\ 43-5,40-42 & 415.63 & 52 & 9 & 17 & 15 & 6 & 0 & 0 & 0 \\ 46, C C(11-13) & 428.58 & 20 & 17 & 22 & 16 & 11 & 9 & 0 & 0 \\ 56-3,80-82 & 489.03 & 4 & 2 & 3 & 4 & 2 & 0 & 78(7) & 0 \\ 50, C C(20-22) & 494.60 & 0 & 2 & 4 & 4 & 2 & 0 & 81(7) & 0 \\ 52-1,23-25 & 504.46 & 11 & 3 & 10 & 8 & 3 & 4 & 58(3) & 0\end{array}$

Hole 616

$\begin{array}{lrrrrrrrrr}2-6,20-22 & 13.72 & 56 & 8 & 12 & 16 & 7 & 1 & 0 & 0 \\ 7-1,30-32 & 53.92 & 38 & 10 & 14 & 18 & 9 & 2 & 6 & 5 \\ 10-1,71-73 & 83.34 & 49 & 10 & 13 & 18 & 6 & 2 & 0 & 2 \\ 17-3,34-36 & 155.36 & 65 & 6 & 9 & 12 & 5 & 2 & 0 & 1 \\ 21-1,46-48 & 190.68 & 49 & 10 & 16 & 16 & 7 & 2 & 0 & 0 \\ 28-1,12-14 & 305.45 & 25 & 16 & 23 & 22 & 6 & 2 & 0 & 6 \\ 33, C C(21-23) & 357.34 & 33 & 14 & 22 & 19 & 9 & 2 & 0 & 0 \\ 34-1,93-95 & 362.64 & 47 & 10 & 16 & 17 & 8 & 2 & 0 & 0\end{array}$

Hole 617

$\begin{array}{lrrrrrrrrr}1-1,90-92 & 0.91 & 45 & 9 & 14 & 19 & 8 & 4 & 0 & 0 \\ 3-6,60-62 & 25.72 & 62 & 5 & 12 & 14 & 4 & 2 & 0 & 0 \\ 5-1,28-30 & 37.09 & 42 & 11 & 15 & 22 & 6 & 1 & 0 & 2 \\ 6-3,50-52 & 49.82 & 45 & 9 & 15 & 19 & 8 & 3 & 0 & 2 \\ 11-1,44-46 & 94.57 & 35 & 11 & 19 & 22 & 8 & 2 & 0 & 2 \\ 15-2,16-18 & 133.88 & 30 & 13 & 21 & 22 & 5 & 2 & 5 & 3\end{array}$

Hole 620

\begin{tabular}{rrrrrrrrrr}
$13-2,60-62$ & 109.93 & 51 & 8 & 12 & 16 & 6 & 2 & 3 & 3 \\
$14-2,60-62$ & 119.13 & 42 & 10 & 13 & 15 & 10 & 4 & 0 & 6 \\
$15-2,60-62$ & 128.73 & 55 & 5 & 8 & 11 & 4 & 1 & 5 & 2 \\
$16-2,60-62$ & 138.33 & 47 & 9 & 15 & 17 & 8 & 4 & 2 & 2 \\
$17-2,60-62$ & 147.92 & 55 & 7 & 10 & 14 & 7 & 4 & 0 & 3 \\
$17-6,60-62$ & 153.92 & 47 & 8 & 13 & 15 & 9 & 3 & 0 & 6 \\
$18-2,60-62$ & 157.52 & 57 & 7 & 10 & 14 & 5 & 1 & 3 & 2 \\
$19-1,60-62$ & 165.63 & 43 & 9 & 13 & 14 & 10 & 5 & 0 & 6 \\
$20-4,60-62$ & 179.73 & 50 & 8 & 10 & 16 & 6 & 2 & 5 & 3 \\
$21-2,60-62$ & 186.13 & 43 & 10 & 15 & 16 & 9 & 2 & 0 & 3 \\
$22-2,60-62$ & 195.52 & 49 & 10 & 13 & 16 & 5 & 1 & 4 & 2 \\
$23-2,59-61$ & 204.91 & 30 & 11 & 21 & 20 & 9 & 3 & 0 & 6 \\
$24-1,60-62$ & 212.92 & 39 & 11 & 15 & 18 & 5 & 2 & 5 & 4 \\
$25-1,50-52$ & 222.31 & 32 & 13 & 19 & 16 & 10 & 3 & 0 & 6 \\
$26-2,60-62$ & 233.42 & 41 & 13 & 17 & 18 & 4 & 2 & 3 & 2 \\
$27-1,60-62$ & 241.52 & 36 & 12 & 17 & 18 & 8 & 3 & 1 & 5 \\
\hline
\end{tabular}

Table 2 (continued).

\begin{tabular}{|c|c|c|c|c|c|c|c|c|c|}
\hline \multirow{2}{*}{$\begin{array}{c}\text { Core-Section } \\
\text { (interval in } \mathrm{cm} \text { ) }\end{array}$} & \multirow{2}{*}{$\begin{array}{l}\text { Sub-bottom } \\
\text { depth }(m)\end{array}$} & \multicolumn{8}{|c|}{ Clay fraction composition $(\%)$} \\
\hline & & Sm & 1 & $\mathrm{Ch}$ & K & Q & $\mathrm{F}$ & $\mathrm{Ca}^{\mathrm{a}}$ & Do \\
\hline \multicolumn{10}{|l|}{ Hole 620 (Cont.) } \\
\hline $28-2,45-47$ & 252.48 & 33 & 14 & 17 & 18 & 5 & 3 & 7 & 4 \\
\hline $29-1,60-62$ & 260.72 & 44 & 13 & 15 & 16 & 6 & 2 & 0 & 5 \\
\hline $30-2,60-62$ & 271.83 & 46 & 11 & 15 & 16 & 7 & 2 & 0 & 4 \\
\hline $31-2,60-62$ & 281.42 & 37 & 14 & 18 & 18 & 7 & 2 & 0 & 5 \\
\hline $32-2,50-52$ & 291.03 & 37 & 13 & 17 & 18 & 4 & 2 & 5 & 3 \\
\hline $33-2,60-62$ & 300.63 & 24 & 15 & 22 & 17 & 8 & 3 & 3 & 8 \\
\hline $33-5,60-62$ & 305.13 & 30 & 15 & 19 & 18 & 4 & 1 & 7 & 5 \\
\hline $35-2,60-62$ & 319.83 & 30 & 15 & 19 & 19 & 7 & 2 & 1 & 7 \\
\hline $36-2,60-62$ & 329.42 & 39 & 13 & 17 & 17 & 4 & 1 & 5 & 3 \\
\hline $37-2,60-62$ & 339.03 & 45 & 10 & 15 & & 5 & 2 & 4 & 5 \\
\hline $38-2,60-62$ & 348.63 & 34 & 16 & 21 & 19 & 3 & 0 & 4 & 4 \\
\hline $40-2,60-62$ & 367.63 & 27 & 15 & 22 & 19 & 6 & 4 & 0 & 9 \\
\hline $41-4,60-62$ & 380.13 & 13 & 13 & 23 & 15 & 8 & 6 & 9 & 14 \\
\hline $42-2,60-62$ & 386.63 & 35 & 9 & 15 & 20 & 11 & 4 & 0 & 7 \\
\hline $43-1,35-37$ & 394.38 & 15 & 12 & 27 & 19 & 4 & 3 & 11 & 9 \\
\hline $44-3,60-62$ & 407.13 & 33 & 9 & 20 & 23 & 11 & 4 & 0 & 0 \\
\hline
\end{tabular}

Hole 621

$\begin{array}{lrrrrrrrrr}4-1,60-62 & 23.33 & 40 & 12 & 16 & 24 & 7 & 2 & 0 & 0 \\ 14-4,60-62 & 91.82 & 37 & 12 & 18 & 25 & 7 & 1 & 0 & 0 \\ 26-1,73-75 & 167.05 & 32 & 11 & 20 & 26 & 8 & 2 & 0 & 2 \\ 27-1,90-92 & 176.82 & 33 & 12 & 19 & 26 & 7 & 3 & 0 & 0 \\ 32-1,90-92 & 202.23 & 29 & 14 & 20 & 19 & 6 & 1 & 8 & 4 \\ 33-2,90-92 & 213.82 & 34 & 15 & 22 & 0 & 0 & 7 & 0 & 0\end{array}$

Hole 622

$\begin{array}{lrrrrrrrrr}4-2,60-62 & 24.83 & 39 & 12 & 17 & 20 & 8 & 2 & 0 & 2 \\ 6-4,60-62 & 46.82 & 26 & 12 & 21 & 29 & 8 & 3 & 0 & 1 \\ 10-3,60-62 & 93.02 & 30 & 13 & 20 & 26 & 7 & 3 & 0 & 1 \\ 12-1,60-62 & 109.13 & 34 & 12 & 19 & 25 & 7 & 2 & 0 & 1 \\ 13-3,60-62 & 115.93 & 27 & 12 & 20 & 30 & 6 & 3 & 0 & 2 \\ 16-1,60-62 & 133.52 & 54 & 7 & 11 & 16 & 9 & 3 & 0 & 0 \\ 16-2,60-62 & 135.02 & 26 & 12 & 23 & 28 & 7 & 2 & 0 & 2 \\ 22-1,60-62 & 175.33 & 61 & 5 & 12 & 13 & 6 & 3 & 0 & 0 \\ 22-2,60-62 & 176.83 & 48 & 11 & 14 & 18 & 5 & 4 & 0 & 0 \\ 23-1,65-67 & 178.38 & 62 & 5 & 11 & 12 & 8 & 2 & 0 & 0 \\ 23-2,65-67 & 179.88 & 37 & 15 & 17 & 20 & 7 & 5 & 0 & 0\end{array}$

Hole 623

$\begin{array}{rrrrrrrrrr}1-1,90-92 & 0.92 & 37 & 10 & 12 & 17 & 16 & 7 & 0 & 0 \\ 1-4,90-92 & 5.43 & 47 & 8 & 12 & 15 & 12 & 7 & 0 & 0 \\ 2-3,60-62 & 10.23 & 53 & 8 & 14 & 15 & 6 & 2 & 0 & 2 \\ 2-6,60-62 & 14.73 & 46 & 8 & 12 & 16 & 8 & 5 & 0 & 5 \\ 3-2,60-62 & 18.33 & 38 & 12 & 18 & 21 & 5 & 4 & 0 & 3 \\ 3-5,60-62 & 22.83 & 58 & 7 & 10 & 14 & 8 & 4 & 0 & 0 \\ 4-3,60-62 & 29.42 & 54 & 8 & 13 & 19 & 4 & 2 & 0 & 1 \\ 5-1,60-62 & 36.03 & 54 & 6 & 10 & 13 & 8 & 4 & 0 & 5 \\ 5-4,60-62 & 40.53 & 50 & 9 & 13 & 16 & 5 & 2 & 1 & 4 \\ 6-2,60-62 & 47.13 & 50 & 6 & 11 & 14 & 11 & 4 & 0 & 4 \\ 6-5,60-62 & 51.63 & 44 & 8 & 13 & 18 & 6 & 3 & 4 & 5 \\ 7-3,60-62 & 58.22 & 65 & 7 & 8 & 11 & 6 & 3 & 0 & 0 \\ 8-2,95-97 & 66.68 & 44 & 9 & 13 & 17 & 6 & 2 & 4 & 5 \\ 9-2,60-62 & 75.93 & 48 & 9 & 13 & 17 & 7 & 3 & 0 & 3 \\ 10-2,25-27 & 85.17 & 46 & 10 & 14 & 18 & 6 & 1 & 1 & 4 \\ 12-2,60-62 & 104.73 & 58 & 7 & 12 & 12 & 6 & 5 & 0 & 0 \\ 14-1,59-61 & 122.01 & 59 & 6 & 11 & 15 & 6 & 3 & 0 & 0 \\ 16-2,60-62 & 142.23 & 57 & 6 & 11 & 15 & 7 & 3 & 0 & 0\end{array}$

Note. Abbreviations are as follows: $\mathrm{Sm}=$ smectite, $\mathrm{I}=$ illite; $\mathrm{Ch}=$ chlorite, $\mathrm{K}=\mathrm{ka}$ olinite, $\mathrm{Q}=$ quartz, $\mathrm{F}=$ feldspar, $\mathrm{Ca}=$ calcite, $\mathrm{Do}=$ dolomite.

a Values in $\mathrm{Ca}$ column in parentheses are for aragonite.

were measured for all the identified minerals and normalized to 100 without introducing any intensity-weighting factors. The percentages given are, therefore, relative percentages only for comparison within the data set.

\section{RESULTS}

\section{Inorganic Geochemistry}

Table 1 shows the bulk inorganic geochemistry for all samples analyzed from Sites 615, 616, 620, 621, and 623 in the Mississippi Fan, along with data from Sites 618 and 619 in the intraslope basins. Since this chapter is 
concerned primarily with the fan sites, no further mention is made of the data from the intraslope basins although the data are included for comparative purposes.

The inorganic geochemistry data indicate relatively little variability both within and between sites (Fig. 2, Table 1). The most notable variations occur in the six samples from the carbonate interval at the bottom of Site 615 that contain between 35 and 41 weight percent $\mathrm{CaO}$ (recalculated to about $62-73$ wt. $\% \mathrm{CaCO}_{3}$ ) and about 1300-2175 ppm Sr, compared to values of 3-5 wt. \% $\mathrm{CaO}$ and 150-200 ppm Sr, more typical of all the other samples. Macroscopic core descriptions and carbonate bomb tests indicate that the section from which these samples came consists of a nannofossil ooze with $75-80 \% \mathrm{CaCO}_{3}$ (see Site 615 chapter, this volume). The rest of the fan sediments consist of clay, mud, silt, sand, and gravel of terrigenous origin (see site chapters, this volume).

A bivariant analysis for the 22 major, minor, and trace elements from the fan sites, excluding the six carbonate samples at the base of Site 615, was used to aid in the interpretation of the mineralogical source of the oxides and elements (Table 3 ). On the basis of all the available mineralogical and geochemical data, the following conclusions may be drawn from this analysis:

1. Most of the major element oxides (e.g., $\mathrm{Al}_{2} \mathrm{O}_{3}$, $\mathrm{FeO}+\mathrm{Fe}_{2} \mathrm{O}_{3}, \mathrm{MgO}$, and $\mathrm{SiO}_{2}$ ) may be accounted for in the various clay minerals. One example of this is shown by the high correlation coefficient of 0.96 between $\mathrm{Al}_{2} \mathrm{O}_{3}$ and $\mathrm{FeO}+\mathrm{Fe}_{2} \mathrm{O}_{3}$.

2. The high correlation coefficients between $\mathrm{K}_{2} \mathrm{O}$, $\mathrm{TiO}_{2}, \mathrm{P}_{2} \mathrm{O}_{5}, \mathrm{Cu}, \mathrm{Co}, \mathrm{Li}$ and $\mathrm{Sc}$, and both $\mathrm{Al}_{2} \mathrm{O}_{3}$ and $\mathrm{FeO}+\mathrm{Fe}_{2} \mathrm{O}_{3}$, suggests that a large proportion of these minor and trace elements are associated with the clay minerals. Also, the high correlation coefficients between most of the base metals suggests that they tend to be associated together, probably within some of the clay minerals.

3. The high absolute abundances of some of the minor and trace elements, such as $\mathrm{TiO}_{2}, \mathrm{Cr}, \mathrm{Cu}$, and $\mathrm{Ni}$, may be partly explained by the relatively large amount of accessory and heavy minerals in the fan sediments (Stow et al., this volume). However, sorption of minor and trace elements within clay mineral lattices probably accounts for most of the absolute abundance levels.

4. Above approximately $100 \mathrm{~m}$ sub-bottom, as seen in Sites 615, 616, and 621 (Figs. 1, 2), the samples show a greater variation in the abundances of the major, minor, and trace elements than below this depth. Some samples in the uppermost $100 \mathrm{~m}$ show larger amounts of the oxides and elements that constitute, and are associated with, the clay minerals. Perhaps this increase in clay mineral content of the sediments in the upper $100 \mathrm{~m}$ reflects the rise in sea level and consequent lesser amounts of coarser grained material being deposited on the Mississippi Fan.

\section{Clay Mineralogy}

Typical X-ray diffraction analyses of the $<4 \mu \mathrm{m}$ fraction of all the samples analyzed showed $30-50 \%$ smec- tites, $15-20 \%$ kaolinite, $12-18 \%$ chlorite, $8-14 \%$ illite, $8-12 \%$ quartz, 3-6\% feldspar (mainly plagioclase), $0.5 \%$ calcite (with rare aragonite), and $0.3 \%$ dolomite (Table 2 ). Thus, smectites are clearly the dominant clay minerals present, although their absolute abundance varies considerably between samples. Kaolinite and chlorite are approximately co-equal and of secondary importance. Illite abundances typically range between 0.5 and 0.7 times that of the kaolinite or chlorite abundance.

Subtle variations in mineralogy between the Mississippi Fan sites are evident. The lower fan sites (615 and 623) contain more quartz and feldspar than the middle fan sites (620 and 621). Also, the middle fan overbank site (620) contains greater amounts of calcite and dolomite than either the middle fan channel (621) or any of the lower fan sites (615 and 623).

Although the clay mineralogy varies between sites, there are no obvious horizontal gradients and no consistent downhole variations. However, subtle differences are apparent at Sites 615 and 616. At these sites, a slight and somewhat erratic downhole decrease in smectite occurs, with corresponding increases in kaolinite, chlorite, and illite. The high calcite content at the base of Site 615 , and at the top of most sites, is entirely facies controlled (Stow et al., this volume).

Sediment facies control on the mineralogy is particularly apparent in three ways. (1) The ooze at the base of Site 615 and the ooze and calcareous mud at the top of each site naturally have large amounts of calcite, minor aragonite, and no dolomite - the latter mineral being absent, perhaps, because the environment is not sufficiently reducing. (2) Calcite and dolomite abundance increases in the finer grained facies, with these minerals normally being absent from coarse-grained silts and sands. (3) The thicker silts and sands tend to contain a smaller total clay fraction, relatively more quartz and feldspar in the clay fraction, and variable (although commonly greater) chlorite:kaolinite ratios than the finer-grained facies.

\section{MODELS FOR DEPOSITION}

The majority of Mississippi Fan DSDP Leg 96 sediments were deposited from turbidity currents and other sediment gravity flows. The inorganic geochemistry and clay mineralogy of these sediments, therefore, reflects resedimentation of the Mississippi, and ancestral Mississippi river and delta sediments, together with the Texas-Louisiana and West Florida Shelf sediments [for a comparison with "average shale" compositions, see Wedepohl (1968)]. Given the extremely rapid rates of sediment accumulation on the fan [typically $5-7 \mathrm{~m} / 1000 \mathrm{yr}$. for the lower fan at Site 615 , nearly $12 \mathrm{~m} / 1000 \mathrm{yr}$. for the middle fan channel fill, and about $11 \mathrm{~m} / 1000 \mathrm{yr}$. for the middle fan overbank Site 620 (Wetzel and Kohl, this volume; site chapters, this volume)], the downhole and between-hole similarity in inorganic geochemistry and clay mineralogy data is predictable. At least during the late Pleistocene, the source of the fan sediments did not change; Pleistocene fan sediments reflect a relatively stable and mineralogically mature provenance (Stow et al., this volume). The samples that contain a large amount 
of $\mathrm{CaCO}_{3}$ are principally composed of calcareous foraminifers and nannofossils and therefore most of the carbonate is of biogenic origin.

Three possible models could explain the inorganic geochemical variations and relative minor- and trace-element enrichment in the analyzed sediment samples: (1) bioconcentration in which marine plankton concentrate minor and trace elements as, for example, organometallic compounds (Leinen and Stakes, 1979); (2) postdepositional geochemical mobility, where migrating pore fluids dissolve and reprecipitate relatively mobile oxides, elements, and other element compounds/phases (Dean et al., 1984), and (3) clay mineral sorption (Tourtelot, 1964). Since DSDP Leg 96 samples do not show high concentrations of organic matter, nor is there any reason to believe that marine plankton or bacteria preferentially concentrated minor and trace elements that have remained after decomposition of such organisms, the bioconcentration model is not favored. Also, the samples do not show any evidence of downhole diagenetic changes (although small sulfide concretions occur in many cores) that could explain the absolute and relative minor- and trace-element concentrations. Therefore, the clay mineral sorption model is favored because of the high correlation coefficients between the major clay mineral oxides and many of the minor and trace elements, with any variability being well explained by changing ratios between quartz, carbonate, and clay minerals.

The inorganic geochemistry and clay mineralogy data show no clear early diagenetic trends either downhole or between sites. However, a scanning electron microscope study has not been carried out on these samples and it is possible that the variability in clay minerals, in part, reflects early diagenesis in the sediments that is not recog- nized by the analytical techniques used in this study. For example, it is possible that the inverse relationship observed between smectites and either dolomite or calcite is a function of smectite authigenesis. Furthermore, much of the dolomite may be early authigenic in origin. Finally, not all the aragonite has inverted to calcite at the base of Site 615 and this may reflect the rapid burial of these sediments.

\section{ACKNOWLEDGMENTS}

A. J. Fleet and I. Jarvis reviewed an earlier draft of this manuscript.

\section{REFERENCES}

Carver, R. E., 1971. Procedures in Sedimentary Petrology: New York (Wiley-Interscience).

Dean, W. E., Arthur, M. A., and Stow, D. A. V., 1984. Origin and geochemistry of Cretaceous deep-sea black shales and multicolored claystones, with emphasis on Deep Sea Drilling Project Site 530, Southern Angola Basin. In Hay, W. W., Sibuet, J.-C., et al., Init. Repts. DSDP, 75: Washington (U.S. Govt. Printing Office), 819-844.

Leinen, M., and Stakes, D., 1979. Metal accumulation rates in the central equatorial Pacific during Cenozoic time. Geol. Soc. Am. Bull., 90:357-375.

Tourtelot, H. A., 1964. Minor-element composition and organic carbon content of marine and nonmarine shales of Late Cretaceous age in the western interior of the United States. Geochim. Cosmochim. Acta, 28:1579-1604.

Walsh, J. N., and Howie, R. A., 1980. An evaluation of the performance of an inductively coupled plasma source spectrometer for the determination of the major and trace element constituents of silicate rocks and minerals. Min. Mag., 43:967-974.

Wedepohl, K. H., 1968. Chemical fractionation in the sedimentary environment. In Ahrems, L. H. (Ed.), Origin and Distribution of Elements: Oxford (Pergamon Press), pp. 999-1016.

Date of Initial Receipt: 21 February 1985

Date of Acceptance: 30 July 1985 

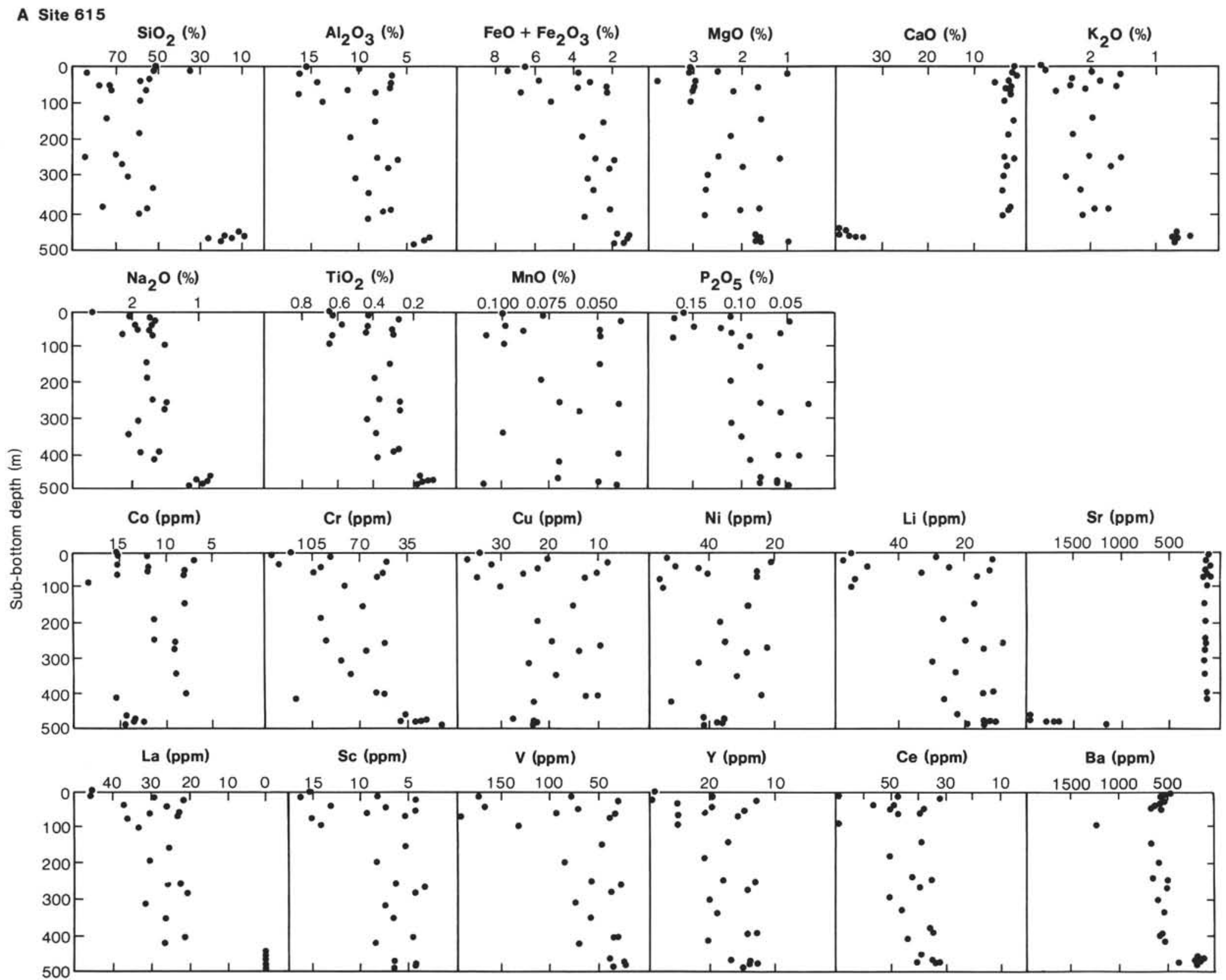

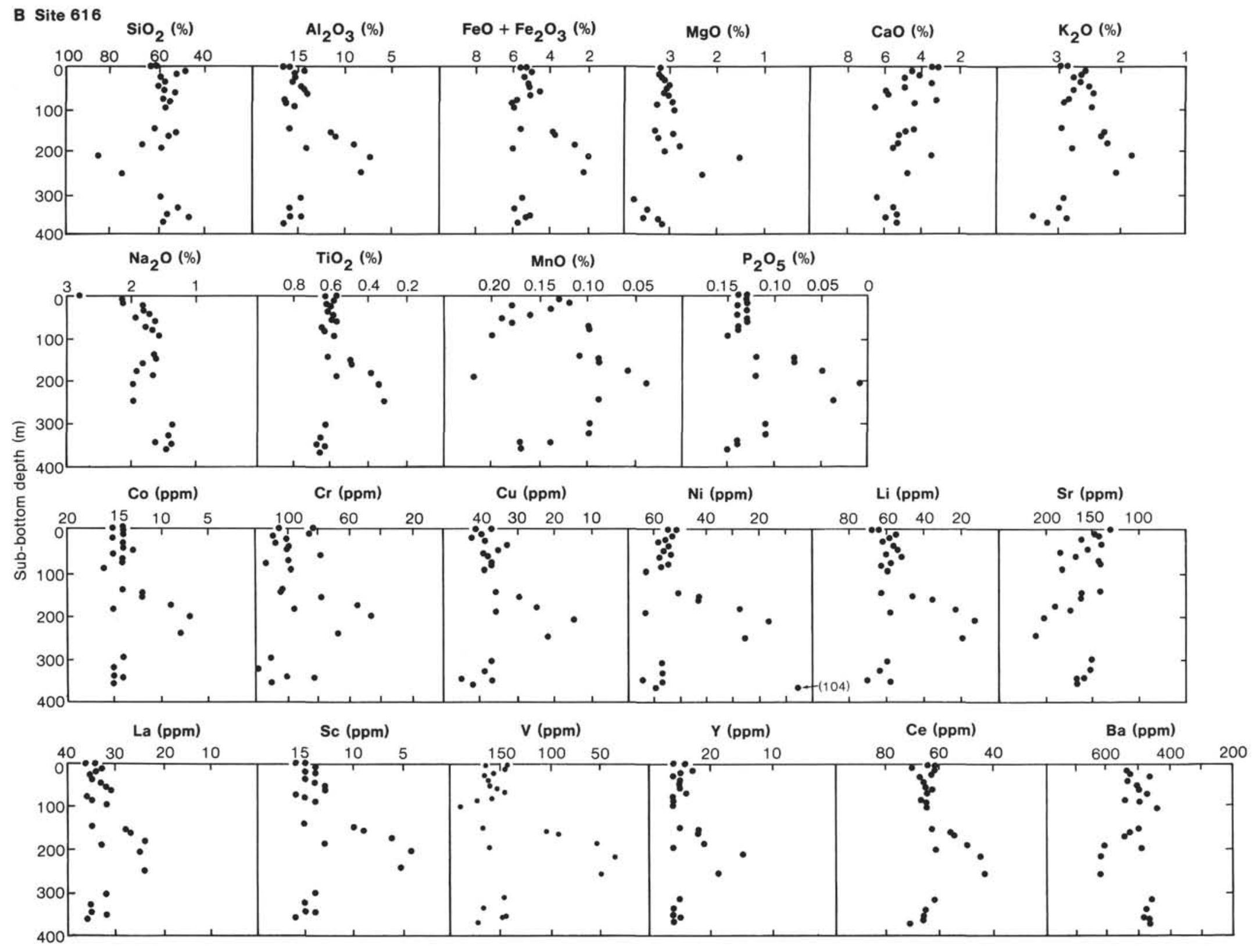

$\pm$

Figure 2. Downhole geochemical trends for various major, minor, and trace elements at sites visited during DSDP Leg 96. See Table 1 for tabulated data. 

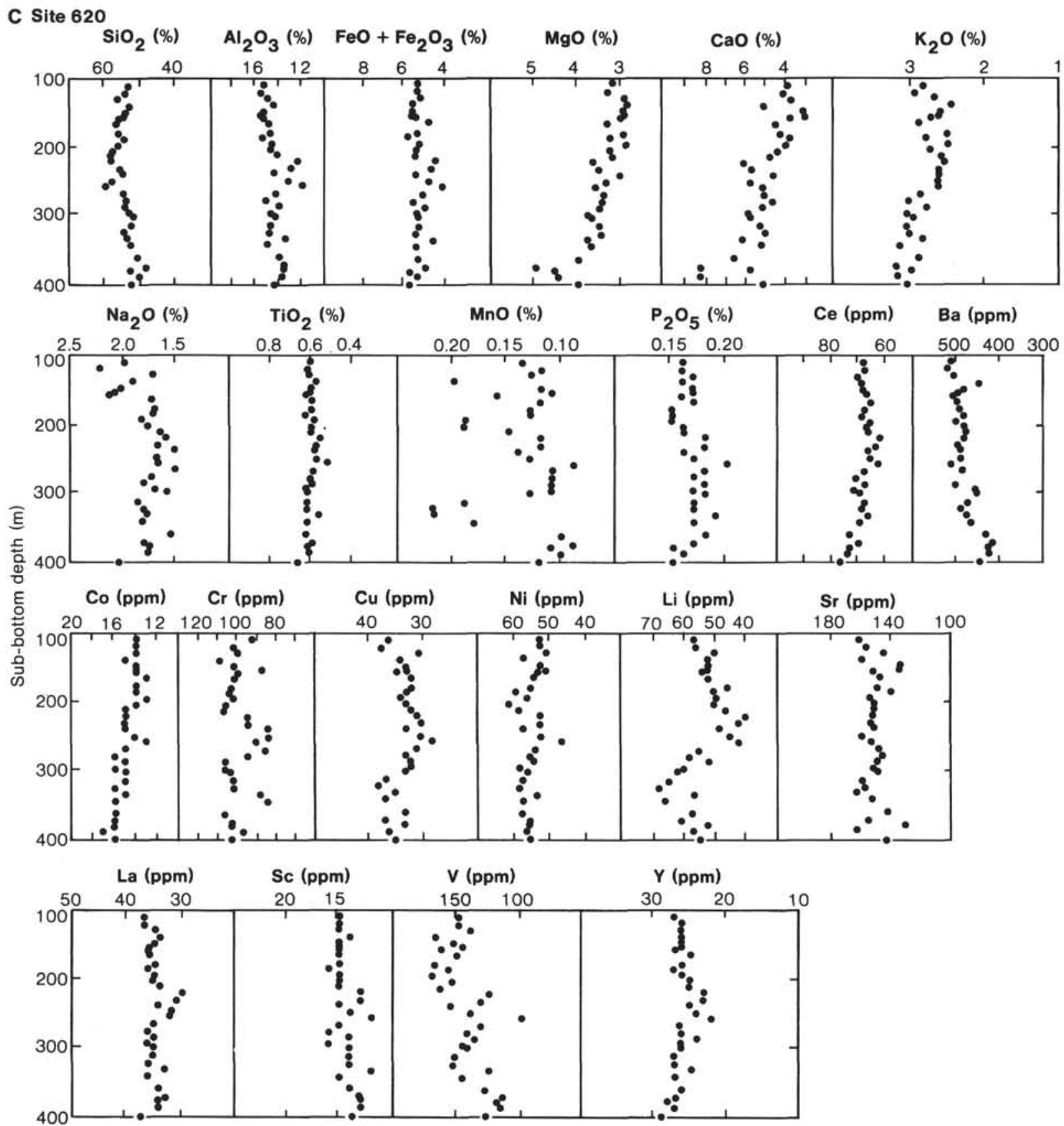


\section{Site 621}

$\mathrm{SiO}_{2}(\%)$

$\mathrm{Al}_{2} \mathrm{O}_{3}(\%)$

$\mathrm{FeO}+\mathrm{Fe}_{2} \mathrm{O}_{3}(\%)$

MgO (\%)

$\mathrm{CaO}(\%)$

$\mathrm{K}_{2} \mathrm{O}(\%)$

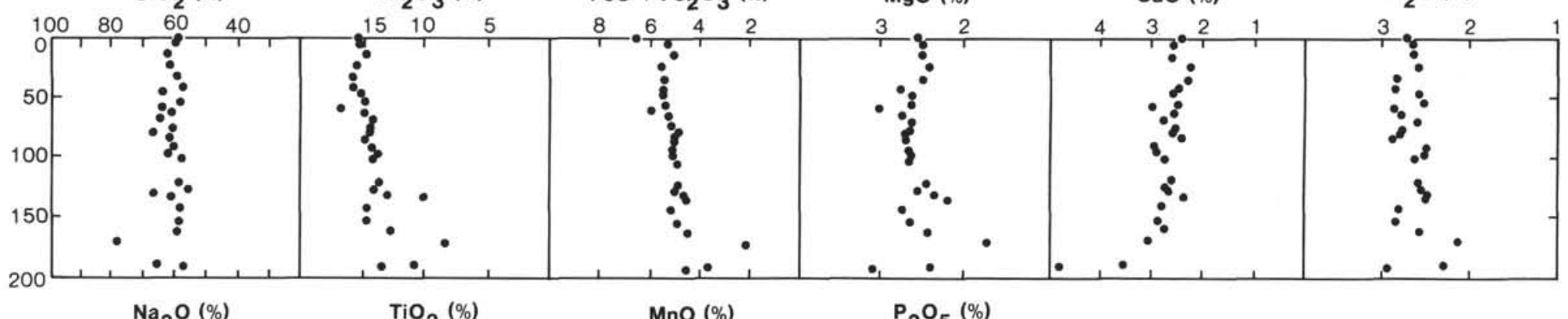

$\mathrm{Na}_{2} \mathrm{O}(\%)$

MnO (\%)

$\mathrm{P}_{2} \mathrm{O}_{5}(\%)$

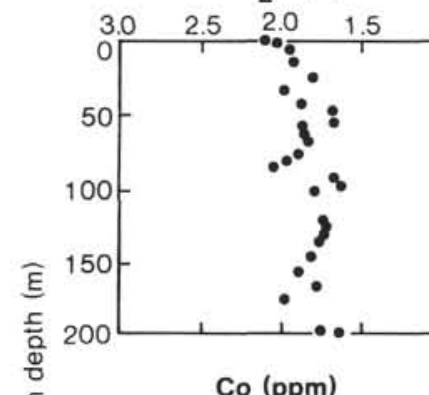

$\mathrm{TiO}_{2}(\%)$
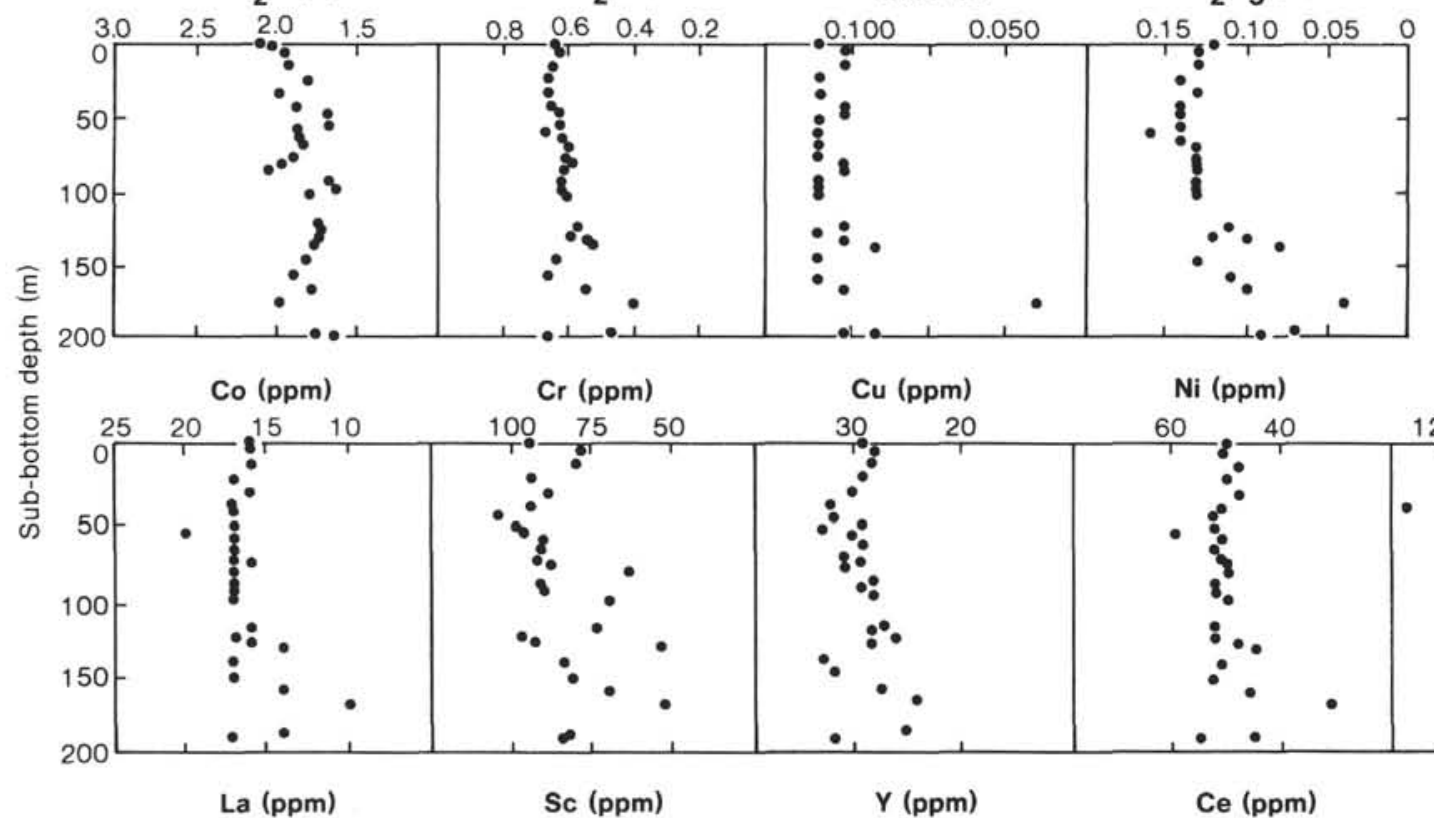

Cr (ppm)

Cu (ppm)

$\mathrm{Ni}(\mathrm{ppm})$

Li (ppm)

Sr (ppm)
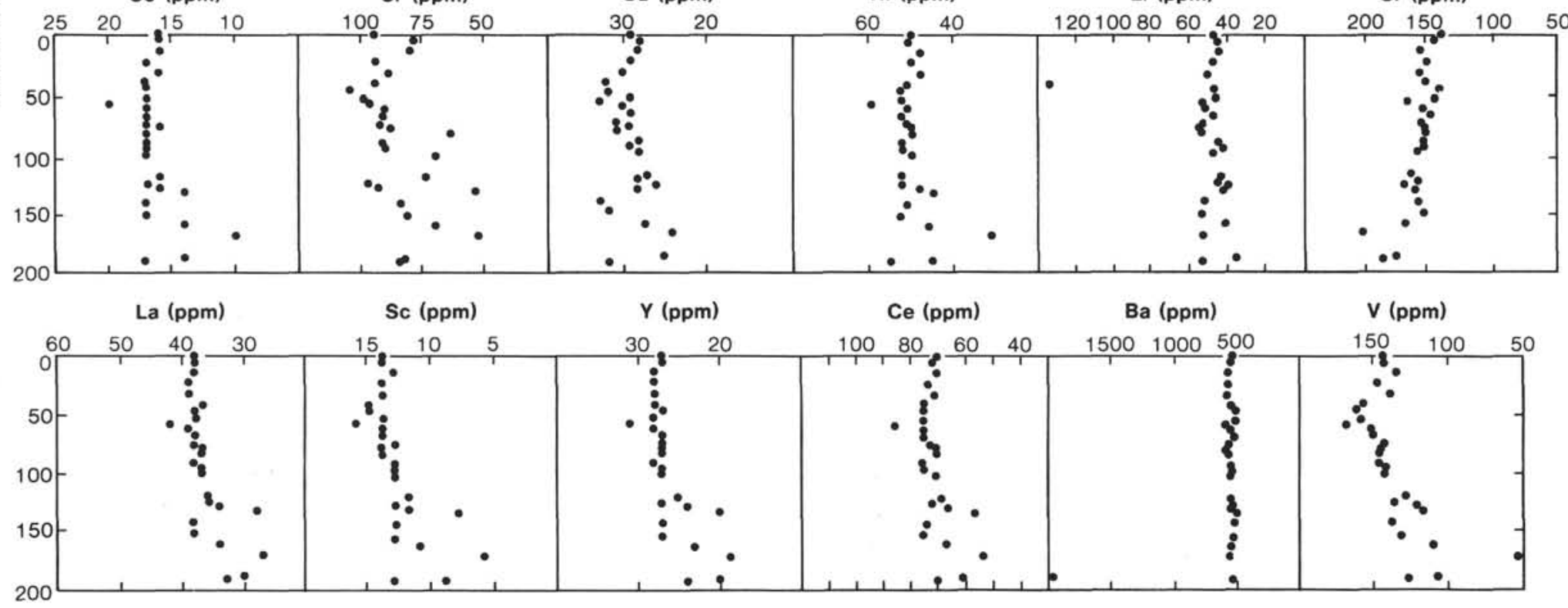

20

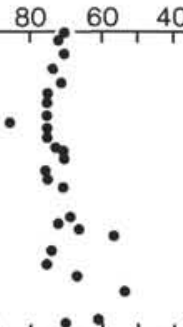



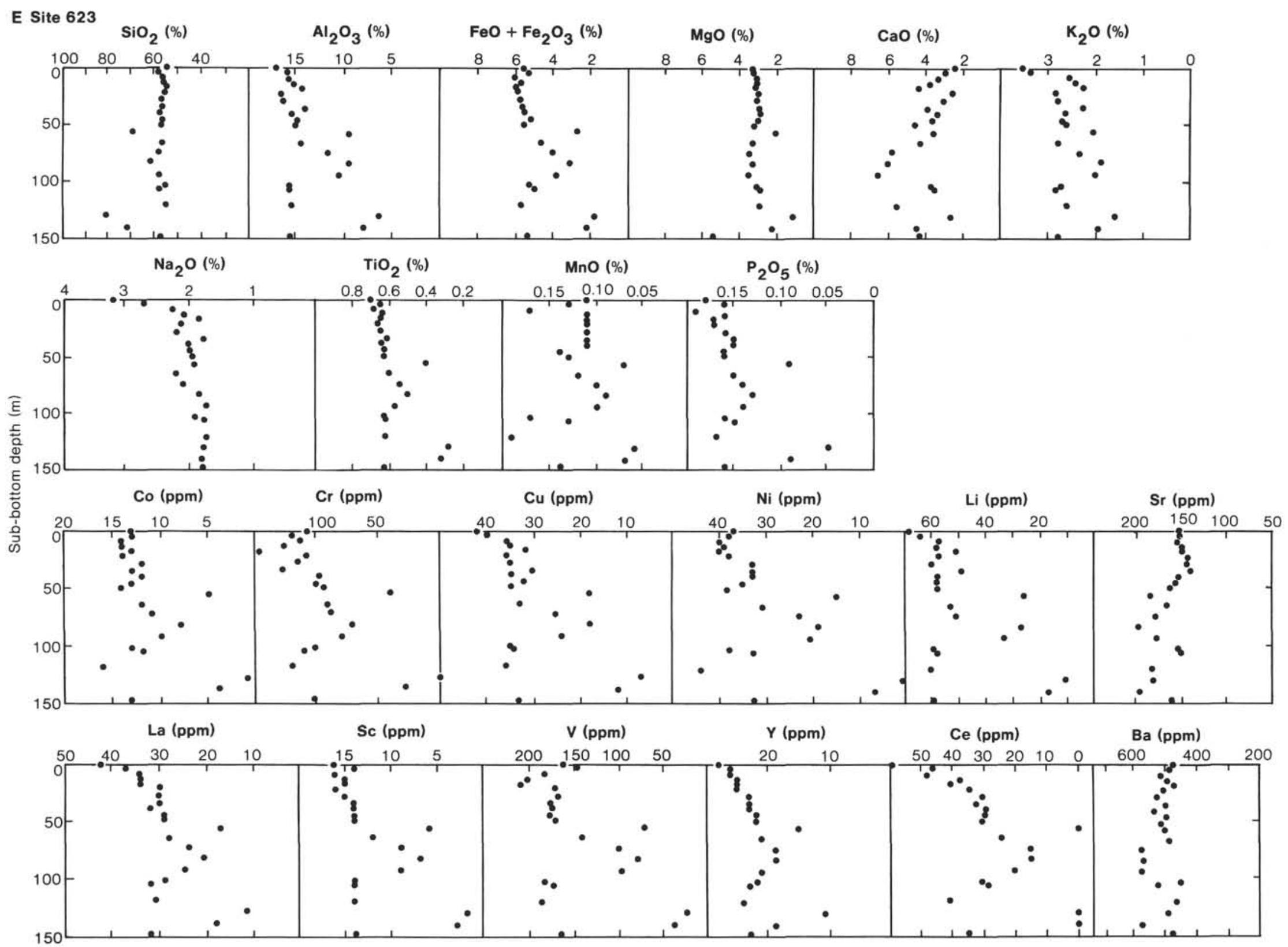

Figure 2 (continued). 
INORGANIC GEOCHEMISTRY AND CLAY MINERALOGY

Table 3. Correlation coefficient table for bivariant analysis of major, minor, and trace elements for Mississippi Fan Sites 615, 616, 620,621 , and 623.

\begin{tabular}{|c|c|c|c|c|c|c|c|c|c|c|c|c|c|c|c|c|c|c|}
\hline & $\mathrm{Al}_{2} \mathrm{O}_{3}$ & $\mathrm{Fe}^{\mathrm{a}}$ & $\mathrm{MgO}$ & $\mathrm{CaO}$ & $\mathrm{Na}_{2} \mathrm{O}$ & $\mathrm{K}_{2} \mathrm{O}$ & $\mathrm{TiO}_{2}$ & $\mathrm{P}_{2} \mathrm{O}_{5}$ & $\mathrm{MnO}$ & $\mathrm{Ba}$ & $\mathrm{Ce}$ & Co & $\mathrm{Cr}$ & $\mathrm{Cu}$ & $\mathrm{Li}$ & $\mathrm{Ni}$ & Sc & $\mathrm{Sr}$ \\
\hline $\mathrm{SiO}_{2}$ & -0.72 & -0.75 & -0.78 & -0.34 & -0.07 & -0.68 & -0.74 & -0.72 & -0.58 & 0.63 & -0.29 & -0.57 & -0.67 & -0.77 & -0.64 & -0.60 & -0.73 & 0.46 \\
\hline $\mathrm{Al}_{2} \mathrm{O}_{3}$ & & 0.96 & 0.58 & -0.10 & 0.20 & 0.85 & 0.95 & 0.84 & 0.63 & -0.53 & 0.43 & 0.74 & 0.71 & 0.90 & 0.84 & 0.69 & 0.97 & -0.49 \\
\hline $\mathrm{Fe}^{\mathrm{a}}$ & & & 0.61 & -0.05 & 0.12 & 0.79 & 0.95 & 0.85 & 0.63 & -0.57 & 0.47 & 0.79 & 0.77 & 0.87 & 0.79 & 0.72 & 0.96 & -0.73 \\
\hline $\mathrm{MgO}$ & & & & 0.63 & 0.04 & 0.71 & 0.65 & 0.60 & 0.45 & -0.57 & 0.28 & 0.53 & 0.61 & 0.72 & 0.59 & 0.59 & 0.62 & -0.28 \\
\hline $\mathrm{CaO}$ & & & & & -0.31 & 0.10 & -0.04 & 0.01 & 0.24 & 0.40 & -0.04 & -0.04 & 0.14 & 0.17 & 0.04 & 0.19 & -0.03 & 0.46 \\
\hline $\mathrm{Na}_{2} \mathrm{O}$ & & & & & & 0.19 & 0.14 & 0.27 & 0.00 & 0.13 & -0.15 & -0.10 & 0.09 & 0.19 & 0.13 & -0.17 & 0.12 & -0.21 \\
\hline $\mathrm{K}_{2} \mathrm{O}$ & & & & & & & 0.84 & 0.65 & 0.50 & -0.57 & 0.53 & 0.72 & 0.55 & 0.86 & 0.82 & 0.69 & 0.84 & -0.51 \\
\hline $\mathrm{TiO}_{2}$ & & & & & & & & 0.85 & 0.60 & -0.53 & 0.44 & 0.80 & 0.71 & 0.87 & 0.82 & 0.68 & 0.95 & -0.72 \\
\hline $\mathrm{P}_{2} \mathrm{O}_{5}$ & & & & & & & & & 0.63 & -0.44 & 0.15 & 0.57 & 0.75 & 0.17 & 0.68 & 0.46 & 0.83 & -0.63 \\
\hline $\mathrm{MnO}$ & & & & & & & & & & -0.51 & 0.24 & 0.47 & 0.51 & 0.70 & 0.58 & 0.56 & 0.62 & -0.16 \\
\hline $\mathrm{Ba}$ & & & & & & & & & & & -0.28 & -0.39 & -0.48 & -0.61 & -0.56 & -0.50 & -0.57 & 0.36 \\
\hline $\mathrm{Ce}$ & & & & & & & & & & & & 0.74 & 0.16 & 0.42 & 0.40 & 0.85 & 0.50 & -0.23 \\
\hline Co & & & & & & & & & & & & & 0.54 & 0.70 & 0.66 & 0.85 & 0.80 & -0.55 \\
\hline $\mathrm{Cr}_{\mathrm{r}}$ & & & & & & & & & & & & & & 0.74 & 0.60 & 0.55 & 0.75 & -0.53 \\
\hline $\mathrm{Cu}$ & & & & & & & & & & & & & & & 0.84 & 0.76 & 0.89 & -0.50 \\
\hline $\mathrm{Li}$ & & & & & & & & & & & & & & & & 0.64 & 0.82 & -0.52 \\
\hline $\mathrm{Ni}$ & & & & & & & & & & & & & & & & & 0.77 & -0.34 \\
\hline Sc & & & & & & & & & & & & & & & & & & -0.70 \\
\hline
\end{tabular}

Note. Carbonates at base of Site 615 are excluded from this analysis using a least-squares regression line. Sample population is 126 unless otherwise stated. Ce plots exclude Sections $623-7-3$, 623-15-1, and 623-16-2; $\mathrm{Cr}$ plots exclude Section 623-15-1; Ba plots exclude Sections 615-12-1 and 621-31-1.

${ }^{\mathrm{a}} \mathrm{Fe}=\mathrm{FeO}+\mathrm{Fe}_{2} \mathrm{O}_{3}$. 